\title{
DYRK1A regulates B cell acute lymphoblastic leukemia through phosphorylation of FOX01 and STAT3
}

\author{
Rahul S. Bhansali, ${ }^{1}$ Malini Rammohan, ${ }^{1}$ Paul Lee, ${ }^{2}$ Anouchka P. Laurent,, ${ }^{3}$ Qiang Wen, ${ }^{1}$ Praveen Suraneni, ${ }^{1}$ Bon Ham Yip, ${ }^{4}$ \\ Yi-Chien Tsai, ${ }^{5}$ Silvia Jenni, ${ }^{5}$ Beat Bornhauser, ${ }^{5}$ Aurélie Siret, ${ }^{3}$ Corinne Fruit, ${ }^{6}$ Alexandra Pacheco-Benichou, ${ }^{6}$ Ethan Harris, ${ }^{7}$ \\ Thierry Besson, ${ }^{6}$ Benjamin J. Thompson, ${ }^{8}$ Young Ah Goo, ${ }^{9}$ Nobuko Hijiya, ${ }^{10}$ Maria Vilenchik, ${ }^{11}$ Shai Izraeli, ${ }^{12}$ Jean-Pierre Bourquin, ${ }^{5}$ \\ Sébastien Malinge, ${ }^{3,13}$ and John D. Crispino, ${ }^{1,4}$ \\ 'Department of Medicine, Division of Hematology/Oncology, Northwestern University, Chicago, Illinois, USA. ${ }^{2}$ Abbvie, North Chicago, Illinois, USA. ${ }^{3}$ INSERM U1170, Gustave Roussy Institute, Villejuif, France. \\ ${ }^{4}$ Division of Experimental Hematology, Department of Hematology, St. Jude Children's Hospital, Memphis, Tennessee, USA. ${ }^{5}$ Department of Pediatric Oncology, Children's Research Centre, University \\ Children's Hospital Zurich, Zurich, Switzerland. ${ }^{6}$ Normandie University, UNIROUEN, Institut National des Sciences Appliquées (INSA) Rouen, CNRS, Chimie Organique et Bioorganique - Réactivité et Analyse \\ (COBRA) UMR 6014, Rouen, France. ${ }^{7}$ College of Medicine, University of Illinois at Chicago, Chicago, Illinois, USA. ${ }^{8}$ Xencor Inc., San Diego, California, USA. ${ }^{9}$ Proteomics Center of Excellence, Northwestern \\ University, Evanston, Illinois, USA. ${ }^{10}$ Division of Pediatric Hematology/Oncology, Columbia University, New York, New York, USA. "'Felicitex Therapeutics Inc., Boston, Massachusetts, USA. ${ }^{12}$ Pediatric \\ Hematology Oncology, Schneider Children's Medical Center, Sackler Faculty of Medicine, Tel Aviv University, Petah Tikva, Israel. ${ }^{13}$ Telethon Kids Institute, Telethon Kids Cancer Centre (TKCC), Nedlands, \\ Western Australia, Australia.
}

\begin{abstract}
DYRK1A is a serine/threonine kinase encoded on human chromosome 21 (HSA21) that has been implicated in several pathologies of Down syndrome (DS), including cognitive deficits and Alzheimer's disease. Although children with DS are predisposed to developing leukemia, especially B cell acute lymphoblastic leukemia (B-ALL), the HSA21 genes that contribute to malignancies remain largely undefined. Here, we report that DYRK1A is overexpressed and required for B-ALL. Genetic and pharmacologic inhibition of DYRK1A decreased leukemic cell expansion and suppressed B-ALL development in vitro and in vivo. Furthermore, we found that FOXO1 and STAT3, transcription factors that are indispensable for B cell development, are critical substrates of DYRK1A. Loss of DYRK1A-mediated FOX01 and STAT3 signaling disrupted DNA damage and ROS regulation, respectively, leading to preferential cell death in leukemic B cells. Thus, we reveal a DYRK1A/FOX01/STAT3 axis that facilitates the development and maintenance of B-ALL.
\end{abstract}

\section{Introduction}

B cell acute lymphoblastic leukemia (B-ALL) is the most commonly diagnosed pediatric cancer, with incidence peaking between the ages of 2 and 5 years (1). The success of multidrug regimens in B-ALL has allowed the overall 5-year event-free survival (EFS) rates to surpass $85 \%$ (2); however, there are still several factors that portend a worse prognosis, such as molecular subtype (e.g., Philadelphia-like [Ph-like] ALL) and age (either less than 1 year or older than 13 years). Notably, children with Down syndrome (DS) have a 27-fold increased risk of developing ALL (DS-ALL) (3), which is almost exclusively of B cell origin (4). DS-ALL blasts harbor extensive genetic heterogeneity; along with the characteristic trisomy 21, somatic alterations leading to $J A K 2$ activation, $C R L F 2$ overexpression, and loss of IKZF1 and PAX5 are common (5). Given that trisomy of chromosome 21 (HSA21) causes DS, there has

\section{Related Commentary: https://doi.org/10.1172/JCl142627}

Authorship note: RSB and MR contributed equally to this work. Conflict of interest: PL and MV are employees of AbbVie Inc. and Felicitex Therapeutics, respectively. BJT is an employee and shareholder of Xencor Inc. NH is a consultant for Novartis and receives research support from Bristol-Myers Squibb. Copyright: (๖) 2021, American Society for Clinical Investigation.

Submitted: December 20, 2019; Accepted: August 11, 2020; Published: January 4, 2021 Reference information: J Clin Invest. 2021;131(1):e135937.

https://doi.org/10.1172/JCl135937. been ongoing investigation into the role of aberrant regulation of HSA21 genes in leukemogenesis (6-10).

DYRK1A, located within the DS critical region (DSCR) of HSA21 (11), encodes dual-specificity tyrosine phosphorylationregulated kinase 1A (DYRK1A). DYRK1A is a serine/threonine kinase that is a member of the evolutionarily conserved CMGC kinase family, which also includes cyclin-dependent kinases (CDKs), Cdc2-like kinases, and other DYRK family members. The role of DYRK1A has been well characterized in neurological pathologies such as autism (12) and Alzheimer's disease (13). These studies have led to the identification of numerous DYRK1A substrates that are involved in essential cellular functions such as apoptosis (14), the cell cycle (15-18), transcription (19), and RNA splicing (20). Despite advances in DYRK1A research in several tissues, there have been very few studies of its role in normal and pathological hematopoiesis.

We previously reported that DYRK1A is a leukemia-promoting gene in acute megakaryoblastic leukemia, which is commonly seen in children with DS, through the dysregulation of nuclear factor of activated $\mathrm{T}$ cells (NFAT) transcription factors (7). We also discovered that DYRK1A is required for normal lymphopoiesis, but dispensable for myeloid cell development (17). This effect on lymphocytes is due to DYRK1A-mediated phosphorylation of cyclin D3, an event that targets it for degradation, thereby promoting cell-cycle exit and subsequent lymphoid maturation. In 
the absence of DYRK1A, B and T cell precursors fail to enter a quiescent state and are unable to progress beyond the large pre-B cell and double-negative thymocyte stages, respectively. Paradoxically, Dyrk1a-deficient pre-B cells also exhibit a proliferative defect without apoptosis, indicating a block in the cell cycle, the mechanism of which has yet to be elucidated. Here, we report that DYRK1A is necessary for the growth of B-ALL cells and represents a therapeutic target in this malignancy. We also demonstrate that FOXO1 and STAT3 are critical substrates of DYRK1A in B lymphopoiesis through the regulation of DNA damage and ROS, respectively. Last, we reveal that DYRK1A, FOXO1, and STAT3 can be effectively targeted in B-ALL through the use of selective and potent small-molecule inhibitors.

\section{Results}

$D Y R K 1 A$ is required for $B-A L L$. Children with DS develop B-ALL at significantly higher rates than do those without DS (3). As these children harbor triplication of DYRK1A due to its localization within the DSCR, and since trisomy of the DSCR promotes leukemogenesis in animal models $(6,7)$, we hypothesized that DYRK1A overexpression would correlate with B cell leukemogenesis. Analysis of the Broad Institute's Cancer Cell Line Encyclopedia (CCLE) (21) revealed that DYRK1A is indeed overexpressed in hematopoietic tumors, and preferentially in acute leukemias, relative to other tumor types (Figure 1A). While a copy number gain of DYRK1A is expectedly seen in ALL and acute myeloid leukemia (AML) cells with abnormalities such as hyperdiploidy (e.g., MHHCALL-2), hypertetraploidy (e.g., TALL-1), and DS (e.g., CMK), cells without gain of HSA21 also have increased DYRK1A expression, indicating that this dysregulation may occur at the transcriptional level (Figure 1B). Moreover, we found that DYRK1A protein levels were increased in both B-ALL cell lines and in patient cells (22) compared with normal human bone marrow mononuclear cells (BMMCs) (Figure 1C). A query of the pediatric cancer data set in St. Jude GenomePaint (23) demonstrated that increased DYRK1A expression is associated with worse EFS in several subtypes of B-ALL, such as hyperdiploid $(\mathrm{HeH})$ and Ph-like ALL (Supplemental Figure 1A; supplemental material available online with this article; https://doi.org/10.1172/JCI135937DS1), underscoring its potential role as a prognostic and therapeutic target.

We previously demonstrated that Dyrk1a-deficient B cells fail to enter quiescence (17), rendering them unable to mature past the large pre-B cell stage. Although this stage is typically characterized by proliferative expansion (24), loss of DYRK1A activity paradoxically impairs cell division, indicating that it is required for both $\mathrm{B}$ cell maturation and proliferation (17). Conversely, overexpression of Dyrk1a alone does not increase clonal proliferation of B-cell precursors in vitro (Supplemental Figure 1, B and C). However, given the upregulation of DYRK1A in B-ALL, we sought to determine whether the gene is required for leukemia cell survival in vivo. We developed a murine model of B-ALL by harvesting lineage-depleted bone marrow cells from mice carrying homozygous (Dyrkla ${ }^{f / f l}$ ) or heterozygous (Dyrk1 $a^{f /+}$ ) conditional knockout alleles of Dyrk1a with or without the Mx1-Cre transgene, transducing these cells with MIGR1-BCR/ABL (p190 isoform), and transplanting them into lethally irradiated recipient mice. Of note, expression of $B C R / A B L$ in murine $\mathrm{CD}^{+} 9^{+}$cells increased DYRK1A protein levels (Figure
1D). Upon detection of $1 \%$ or more $\mathrm{CD} 19^{+} \mathrm{GFP}^{+}$cells in peripheral blood, we initiated treatment with polyI:polyC (pI:pC) to induce excision of Dyrk1a (Figure 1E). Both homozygous and heterozygous deletion of Dyrk1a reduced the leukemic burden in murine bone marrow and spleens and conferred a highly significant survival benefit (Figure 1, F-I, and Supplemental Figure 1D). We found that the animals that succumbed to leukemia harbored B-ALL cells that escaped Dyrk1a excision (Supplemental Figure 1E). Note that control animals with Mx1-Cre and a WT Dyrk1a locus treated with pI:pC succumbed to p190 BCR-ABL-induced B-ALL at the same rate as those of the Dyrk1a $a^{f / f l}$ genotype lacking Mx1-Cre (Supplemental Figure 1, F and G). Thus, while Dyrk1a was not sufficient to drive leukemic transformation in vitro, it was necessary for B-ALL maintenance in vivo. Furthermore, since heterozygous loss of Dyr$k 1 a$ has no observable effect on normal hematopoiesis (17), there appears to be a therapeutic window during which DYRK1A inhibition would selectively target leukemia cells.

$B-A L L$ cells are sensitive to DYRK1A pharmacologic inhibition. Studies have indicated that DYRK1A can perform several functions independent of its kinase activity, such as regulation of chromatin remodeling (25). To determine whether pre-B cell and B-ALL dependence on DYRK1A requires its kinase activity, we mutated the lysine 188 residue of its ATP-binding domain to arginine (K188R), thereby rendering it catalytically inactive. This mutant allele has been previously characterized to have minimal kinase activity and acts as a dominant-negative $(26,27)$. We observed that pre-B cells overexpressing Dyrk1 ${ }^{\mathrm{K} 188 \mathrm{R}}$ completed fewer cell divisions than did those transduced with an empty vector 48 and 72 hours after transduction (Figure 2, A and B), suggesting that its role in B cell proliferation requires its kinase function. Similarly, overexpression of Dyrk1 $a^{\mathrm{K} 188 \mathrm{R}}$ in MHH-CALL- 4 and MUTZ- 5 cells reduced proliferation compared with empty vector-transduced cells (Figure 2C and Supplemental Figure 2A). Notably, overexpression of Dyrk1a $a^{\mathrm{WT}}$ in MHH-CALL-4 and MUTZ- 5 cells did not significantly alter their growth kinetics, possibly indicating that its phenotypic effect had already been elicited by high levels of DYR$K 1 A$ expression in these cell lines.

We next analyzed the effect of DYRK1A inhibition using the small-molecule inhibitor EHT 1610 (28-30). EHT 1610 is a potent and selective small-molecule inhibitor of DYRK1A with known biological activity (31). We previously demonstrated that EHT 1610 exposure recapitulated features of the Dyrk1a knockout such as the loss of pre-B cell colony formation in vitro, without an effect on myelopoiesis or erythropoiesis (17). As seen in murine pre-B cells, treatment of B-ALL cell lines in vitro with EHT 1610 diminished cyclin D3 Thr283 phosphorylation (Figure 2D). EHT 1610 also shifted the cell-cycle distribution of each B-ALL cell that we tested $(n=7)$, with fewer cells in $G_{0}$ and accumulating instead in the $\mathrm{S}-\mathrm{G}_{2}-\mathrm{M}$ phases (Figure 2, E and F, and Supplemental Figure $2 \mathrm{~B})$. We confirmed by DYRK1A-knockdown experiments that these phenotypes were due to the on-target activity of EHT 1610 (Supplemental Figure 2, C-E).

We next found that treatment of B-ALL cell lines and patient-derived xenograft (PDX) B-ALL cells with EHT 1610 resulted in a dose-dependent decrease in cell numbers (Figure $2 \mathrm{G}$, Supplemental Figure 3, and Supplemental Table 1), underscoring the proliferative defect seen when DYRK1A is lost in both WT and 
A

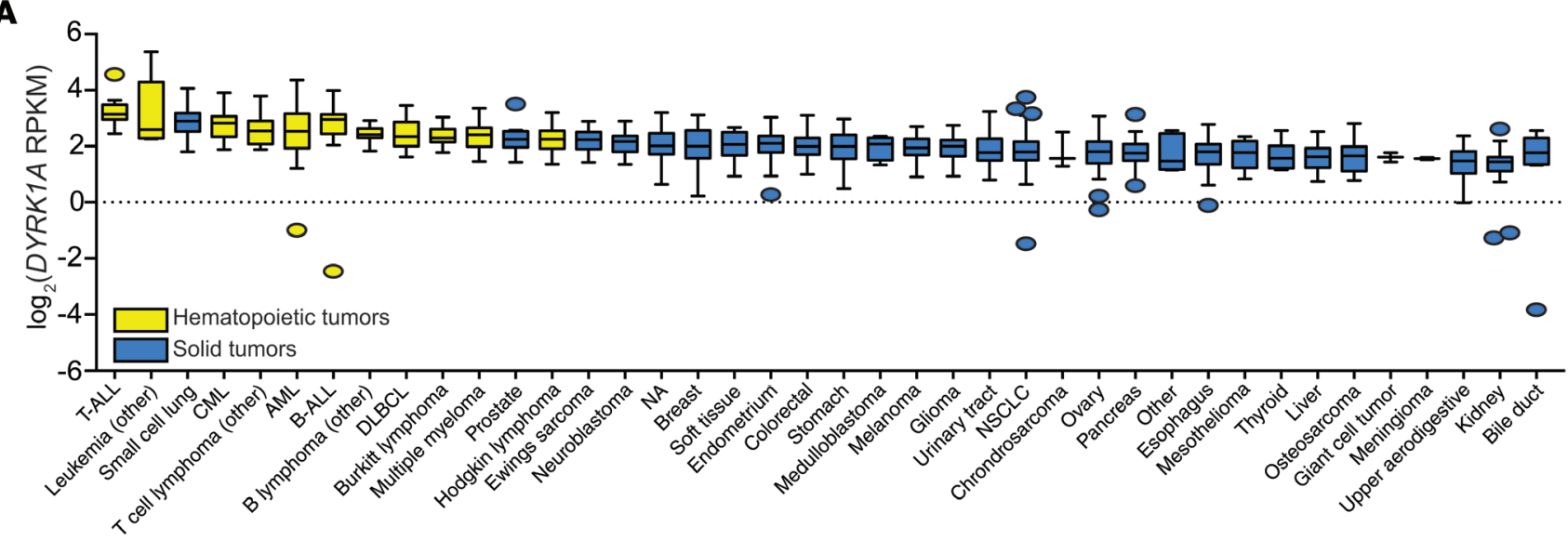

B

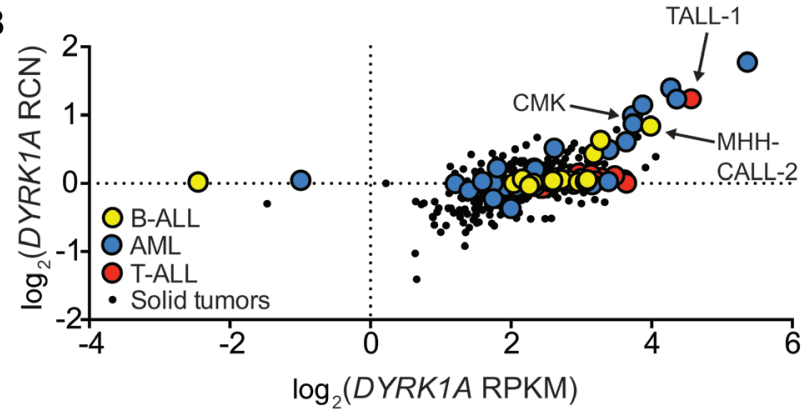

D

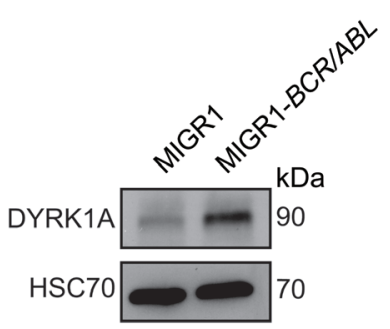

E

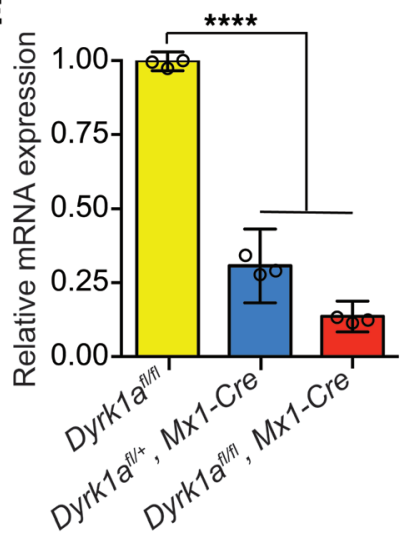

H

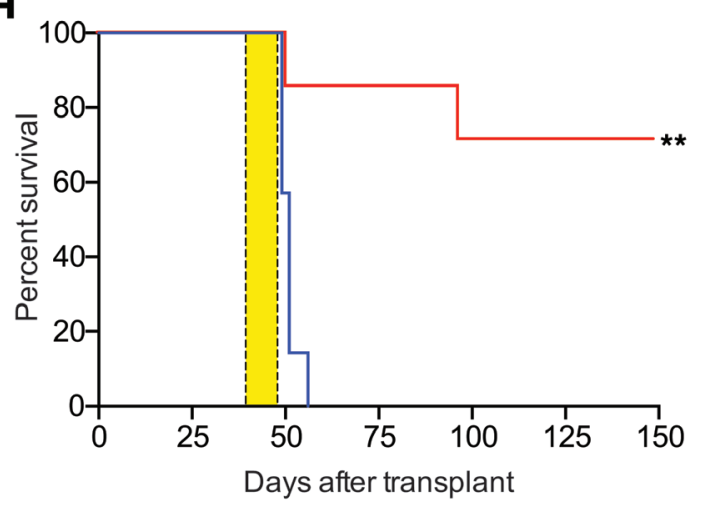

$-D y r k 1 a^{t / / 7}(n=7)$

- Dyrk1a ${ }^{t / 7}$, Mx1-Cre $(P=0.0045, n=7)$

[-] Duration of pl:pC treatment

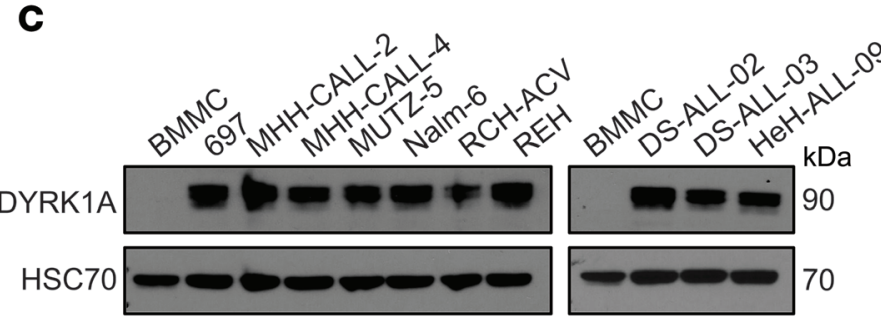

$\mathbf{F}$

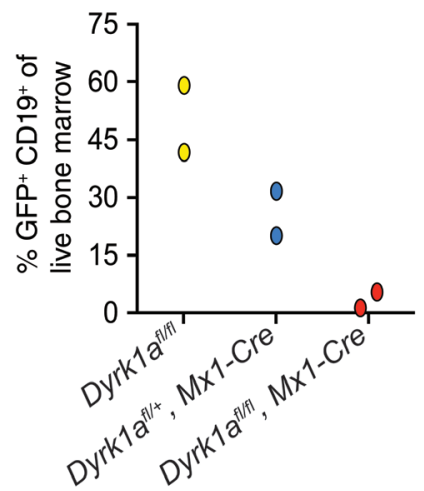

G

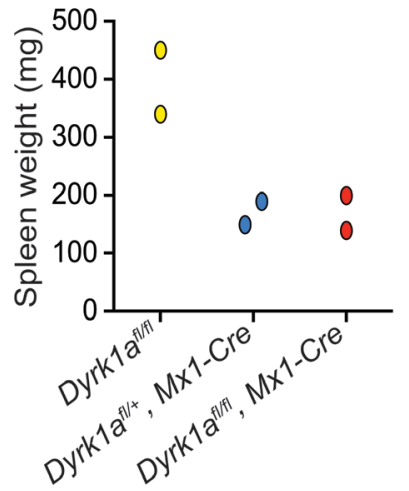


Figure 1. DYRK1A is required for B-ALL. (A) DYRK1A mRNA expression (reads per kb per million mapped reads [RPKM]) distribution across cell lines from the Broad Institute's CCLE, ordered by the median DYRK1A expression level (dotted line), the IQR (box), and up to 1.5 times the IQR (bars). NA, not assigned lineage. (B) DYRK1A mRNA expression versus the relative copy number (RCN) compared across B-ALL $(n=16)$, T-ALL $(n=16)$, AML $(n=35)$, and solid tumors (NSCLC $[n=122]$ and small-cell lung cancer [ $n=50]$, colorectal [ $n=57]$, breast $[n=57]$, and prostate $[n=8])$ from the CCLE. Dots represent individual cell lines. Arrows indicate specific leukemic cell lines with chromosome 21 aneuploidy. (C) Western blot showing total DYRK1A protein in B-ALL cell lines (MHH-CALL-4, MUTZ-5, MHH-CALL-2, REH, RCH-ACV, 697, Nalm-6), patient samples (DS-ALL-02, DS-ALL-03, and HeH-ALL-09), and human BMMCs. Data are from the same gel as in Figure 5C, which was separately probed for FOXO1. Data are representative of 3 independent experiments. (D) Western blot showing total DYRK1A protein in primary murine $C D 19^{+}$cells expressing $B C R-A B L$ (p190). Data are representative of 3 independent experiments. (E-G) Lethally irradiated mice were transplanted with BCR-ABL (p190) B-ALL cells from the bone marrow of Dyrk1a $a^{f / f l}$ or Dyrk1 $a^{f /+}$ mice, with or without $M \times 1-C r e$, and then treated with pl:pC for 2 weeks ( $n=2$ per cohort). Data were analyzed 1 week after completion of $\mathrm{pl}: \mathrm{pC}$ treatment. (E) Dyrk1a mRNA expression following quantitative reverse transcription PCR (qRT-PCR) of sorted CD19+CFP+ ${ }^{+}$cells from murine bone marrow. Data indicate the mean \pm SD (from triplicate wells of a representative sample). (F) Percentage of CD19+GFP+ cells in live bone marrow of individual mice. (G) Spleen weights of individual mice. (H and I) Kaplan-Meier analysis of mice transplanted with BCR-ABL (p190) B-ALL cells from the bone marrow of $D y r k 1 a^{f / f l}(\mathbf{H})$ or $D y r k 1 a^{f /++}(\mathbf{I})$ mice with (red line) or without (blue line) Mx1-Cre after pl:pC injection for 2 weeks (yellow box). $P$ values and sample sizes ( $n$ ) are shown in the key. Data are representative of 2 independent transplantation experiments. Significance was determined by ANOVA with post hoc Bonferroni's correction (E) or logrank (Mantel-Cox) test ( $\mathbf{H}$ and $\mathbf{I}){ }^{* *} P<0.01$ and ${ }^{* * * *} P<0.0001$.

malignant B cells. The effect of DYRK1A inhibition was most striking in MHH-CALL-4 and MUTZ-5 cells, as well as in PDX cells harboring gain of HSA21 (DS-ALL-02 and HeH-ALL-09) (22), in which the $\mathrm{IC}_{50}$ values were in the high nanomolar range. Moreover, compared with previously published DYRK1 inhibitors, including harmine (32) and INDY (33), EHT 1610 displayed more potent activity in B-ALL, T cell ALL (T-ALL), and, particularly, DS-ALL samples (Figure $2 \mathrm{H}$ and Supplemental Table 2). This preferential activity against DS-ALL suggests an increased dependence on DYRK1A in cells with chromosome 21 aneuploidy. Importantly, we also found that EHT 1610 had activity in vitro against high-risk and very high-risk (HR/VHR) ALL, consistent with a dependence on DYRK1A in these poor prognostic subtypes.

B-ALL is typically treated using a multiagent chemotherapeutic regimen of cytotoxic agents that target the cell cycle and proliferation; however, leukemic cells can develop resistance to these agents by escaping into quiescence $(34,35)$. Since DYRK1A inhibition reduces B-ALL cellular quiescence, we hypothesized that EHT 1610 would synergize with conventional chemotherapy. Therefore, we treated B-ALL cell lines that were less sensitive to EHT 1610, including REH, RCH-ACV, Nalm-6, and 697, with 3 commonly used chemotherapy drugs - dexamethasone, cytarabine, and methotrexate - in combination with EHT 1610 at multiples of $\mathrm{IC}_{50}$ values (Supplemental Table 3). Methotrexate treatment was omitted for $\mathrm{REH}$, as its use as a single agent had a subnanomolar $\mathrm{IC}_{50}$. We found that all 3 agents synergized with EHT 1610 in these cell lines (Figure 2I and Supplemental Table 4).
DYRK1A regulates multiple pathways in pre-B cells. We next performed parallel phosphoproteomic experiments to identify substrates of DYRK1A in pre-B cells that could potentially provide insights into the impaired cell growth phenotype. We first used global phosphoproteomics to identify pathways regulated by DYRK1A kinase activity in primary murine pre-B cells (Supplemental Figure $4 \mathrm{~A}$ ). Using a 1.5-fold change threshold, we identified 438 unique dephosphorylated peptides and 272 unique hyperphosphorylated peptides after 2 hours of EHT 1610 treatment (Supplemental Figure 4B). Importantly, the Thr283 residue of cyclin D3 was significantly dephosphorylated with DYRK1A inhibition, consistent with our previous study (17). Using the STRING database (36), we visualized the interactomes of both dephosphorylated and hyperphosphorylated protein data sets and separated them into 5 functionally related groups using KMEANS clustering (Supplemental Figure 4, C and D). We also identified upregulated KEGG annotations (37-39) and found that DYRK1A inhibition affected several pathways such as those involved in the cell cycle, the spliceosome, and transcriptional misregulation in cancer (Figure 3A).

We then leveraged a kinase assay-based phosphoproteomic study to delineate DYRK1A substrates that govern the pathways mentioned above (Supplemental Figure 4E). In this experiment, we dephosphorylated murine pre-B cell lysates with $\lambda$ protein phosphatase and incubated them with recombinant human DYRK1A and ATP to identify peptides that showed increased phosphorylation compared with reactions lacking DYRK1A. Endogenous kinase activity was blocked before re-addition of DYRK1A by treating lysates with $5^{\prime}$-(4-fluorosulfonylbenzonyl)adenosine (FSBA), an irreversible pan-kinase inhibitor, and digesting them with Lys-C protease. This study identified 513 unique peptides with increased phosphorylation compared with the control, including peptides for DYRK1A and human NFATc1, which was spiked in as a positive control. Of these unique phosphorylated peptides, 109 overlapped with the dephosphorylated peptides from our global phosphoproteomics assay (Figure 3, B and C, Supplemental Figure 4F, and Supplemental Table 5) and were used to individually validate bona fide substrates, such as cyclin D3. Notably, our compiled group of peptides included other previously demonstrated DYRK1A substrates such as FOXO1 (40) and STAT3 (41). Gene Ontology (GO) biological process $(42,43)$ analysis of the overlapping protein set revealed enrichment in a variety of annotations that were segregated into RNA processing/splicing/translation, transcriptional regulation/chromatin modification, cell cycle/cell division/cell organization, cell differentiation/development, and cellular metabolic processing/biosynthesis, among others (Figure 3D and Supplemental Table 6). The diversity of enriched annotations dependent on DYRK1A activity underscores its role as a critical regulator of B cell physiology. In particular, the abundance of proteins found to be involved in transcriptional regulation, the cell cycle, and cell differentiation reflects the influence of tightly regulated transcriptional networks in lymphopoiesis (24).

DYRK1A regulates the DNA damage response through phosphorylation of its substrate FOXO1. One candidate DYRK1A substrate identified by our combined phosphoproteomics approach was the transcription factor FOXO1, which we found to be phosphorylated at Ser326 (homologous to human Ser329). We performed an in vitro kinase assay, which validated that DYRK1A phosphor- 

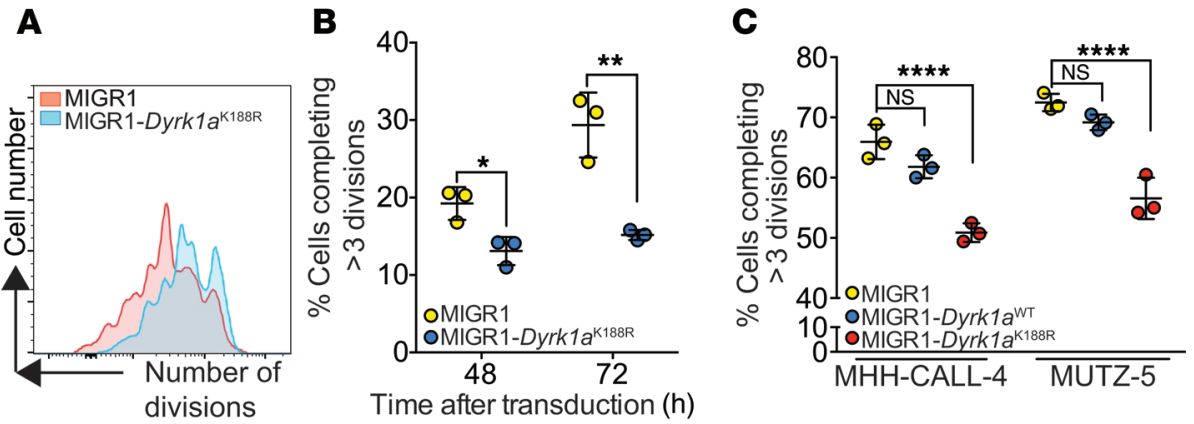

D

E
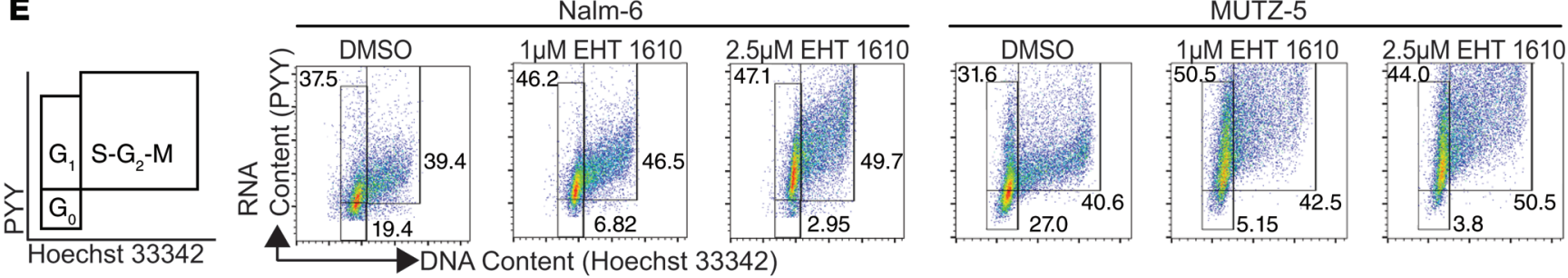

$\mathbf{F}$

F $\square G_{0} \quad \square G_{1} \quad \square S-G_{2}-M$

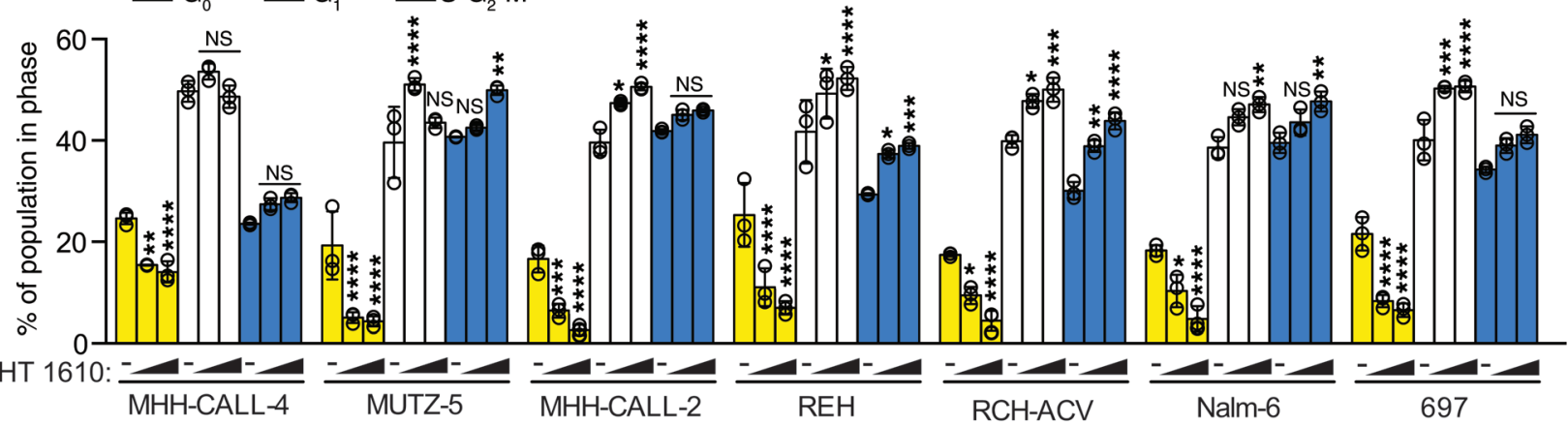

G

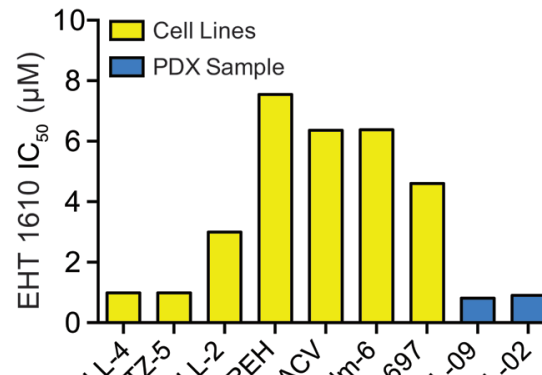

H

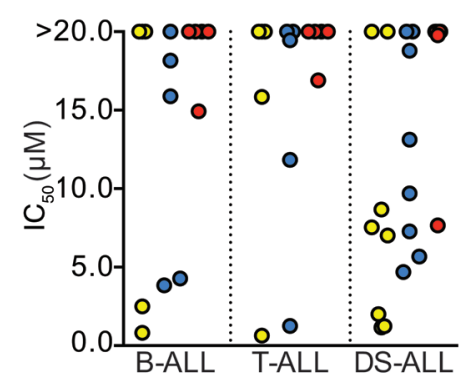

EHT $1610(\mu \mathrm{M}): \begin{array}{llll}0 & 2.5 & 5 & 10 \\ \mathrm{kDa} & \mathrm{k} & & \end{array}$

p-Cyclin D3

(Thr283)

Cyclin D3 $=-24$

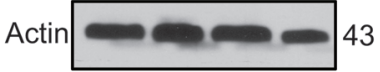

MUTZ-5

$1 \mu \mathrm{M}$ EHT $1610 \quad 2.5 \mu \mathrm{M}$ EHT 1610
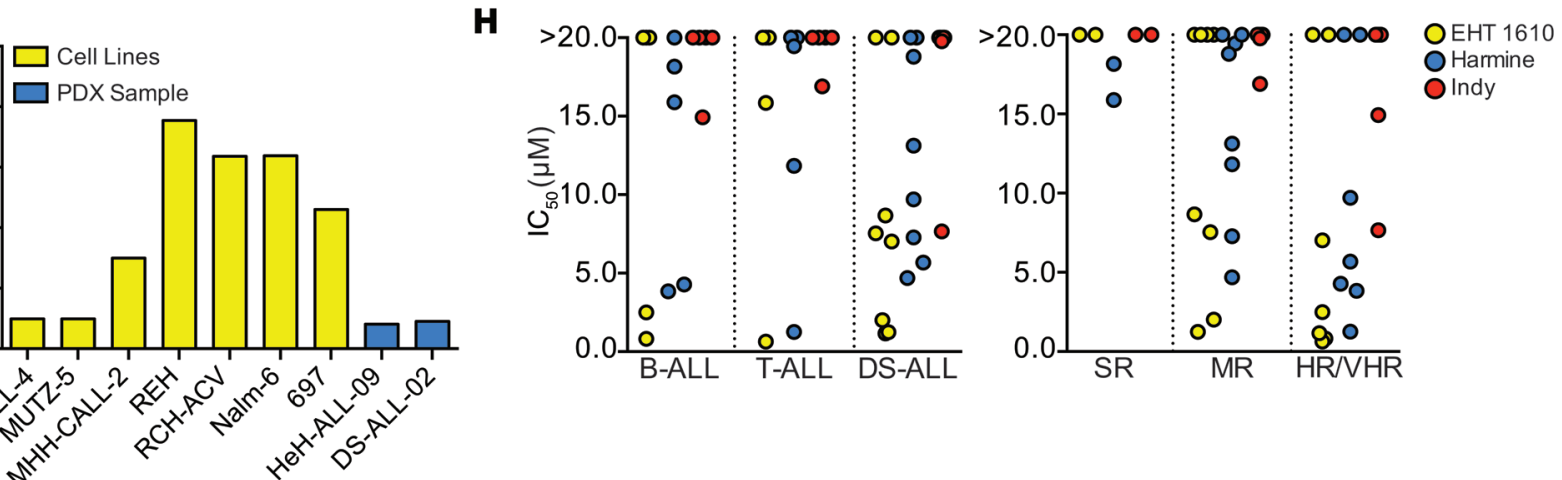

\section{EHT 1610: +}

Dex:

MTX:

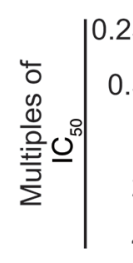

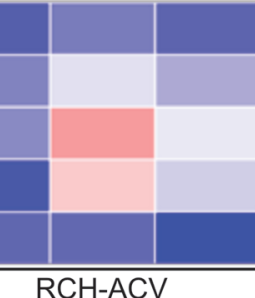

$\mathrm{RCH}-\mathrm{ACV}$

Nalm-6

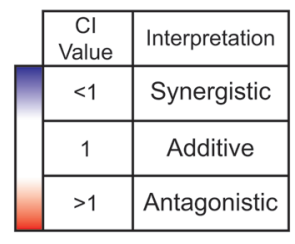


Figure 2. Inhibition of DYRK1A with EHT 1610 impairs B-ALL cell growth. (A) Representative flow cytometric plot of a CellTrace Violet dye dilution assay in $\mathrm{GFP}^{+}$cultured pre-B cells transduced with MICR1 or MIGR1-Dyrk1 $a^{\mathrm{K} 188 R}$. (B) Quantification of the CellTrace Violet dye dilution assay data shown in $\mathbf{A}$ (48 and 72 hours after transduction). Data indicate the mean \pm SD. (C) Quantification of the CellTrace Violet dye dilution assay data shown in Supplemental Figure 2A (120 hours after transduction). Data indicate the mean $\pm \mathrm{SD}$. (D) Western blot showing $\mathrm{p}$-cyclin D3 (Thr283) and total cyclin D3 protein in MHH-CALL-4 cells after 5 hours of treatment with EHT 1610. (E) Representative flow cytometric plots of DNA versus RNA content in Nalm- 6 and MUTZ- 5 cells after a 48-hour treatment with EHT 1610. Numbers indicate the percentage of cells in each gate. (F) Cell-cycle phase distribution based on the gating in $\mathbf{E}$ and Supplemental Figure 2B. Data indicate the mean $\pm S D$. (C) $I_{50}$ values of B-ALL cell lines and patient samples treated with EHT 1610 for 72 or 96 hours based on the data in Supplemental Figure 3. (H) IC ${ }_{50}$ values for patient samples treated with the DYRK1A inhibitors EHT 1610, harmine, or INDY. Dots represent the mean of 3 biological replicates of each patient sample. SR, standard risk; MR, medium risk; HR/VHR, high risk/very high risk. (I) Heatmap of $\mathrm{Cl}$ values when combining EHT 1610 with dexamethasone (Dex), cytarabine (AraC), or methotrexate (MTX) at the multiples of $\mathrm{IC}_{50}$ values indicated in Supplemental Table 3. $n=3$ biological replicates $(\mathbf{A}-\mathbf{I})$. Significance was determined by unpaired $t$ test $(\mathbf{B})$ or ANOVA with post hoc Bonferroni's correction ( $\mathbf{C}$ and $\mathbf{F}$ ). For $\mathbf{F}$, values were compared with DMSO within each cell line. ${ }^{*} P<0.05$, ${ }^{* *} P<0.01,{ }^{* *} P<0.001$, and ****P $<0.0001$.

ylates this residue (Figure 4A). We also observed diminished Ser326 phosphorylation in primary murine pre-B cells with both EHT 1610 treatment and Dyrk1a knockout (Figure 4, B and C). FOXO1 is of particular interest, because it is a known regulator of B lymphopoiesis (44) and is involved in controlling the $\mathrm{G}_{1}-\mathrm{S}$ and $\mathrm{G}_{2}-\mathrm{M}$ checkpoints (45). FOXO1 is canonically regulated by PI3K/ AKT pathway phosphorylation of Thr24, Ser256, and Ser319, which sequesters FOXO1 in the cytoplasm and targets it for degradation $(45,46)$. To determine whether FOXO1 stability is affected by DYRK1A-mediated phosphorylation, we performed a cycloheximide pulse-chase assay and observed increased protein stability upon EHT 1610 treatment (Supplemental Figure 5A). This result suggests that DYRK1A, like PI3K/AKT signaling, regulates FOXO1 through protein destabilization, although by phosphorylation of an alternate site. Of note, our previous study indicated that PI3K/AKT signaling remained intact following DYRK1A inhibition (17), indicating that this phenotype is probably independent of the PI3K/AKT pathway. To elucidate the mechanism by which FOXO1 protein is stabilized in the context of DYRK1A inhibition, we transiently transfected primary murine pre-B cells with WT, phosphomimetic (S329E), and phosphodeficient (S329A) human alleles of FOXO1 fused to GFP. Treatment of WT FOXO1-expressing cells with EHT 1610 altered the subcellular localization, such that it was present within the nucleus despite cytokine stimulation of the IL-7 receptor, which normally activates PI3K/AKT signaling. This nuclear accumulation was seen with the phosphodeficient, but not the phosphomimetic, alleles of FOXO1 (Figure 4D), which is consistent with previous studies (40).

Our previous finding that Dyrk1a-deficient pre-B cells fail to proliferate despite $\mathrm{G}_{1}-\mathrm{S}$ progression due to cyclin D3 stabilization suggested that there may be a distal cell-cycle delay caused by aberrant regulation of a second substrate (17). Indeed, pre-B cells treated with EHT 1610 exhibited a nearly 2-fold increase in $4 \mathrm{~N}$ cells with phosphorylated CDK1 (p-CDK1) without any significant change in dephosphorylated CDK1, indicating that these cells accumulate in early $\mathrm{G}_{2}-\mathrm{M}$, since dephosphorylation of CDK1 is required for entry into mitosis (Figure 4, E and F). Given that FOXO1 regulation of the cell cycle is known to occur at both the $G_{1}-S$ and $G_{2}-M$ checkpoints (45), its dysregulation is a logical explanation for the dual effect of DYRK1A on the cell cycle. At the $\mathrm{G}_{2}-\mathrm{M}$ interface, FOXO1 acts as a DNA damage sensor and delays cell-cycle progression to allow for repair or apoptosis through both P53-dependent and -independent pathways (47). Since loss of DYRK1A activity leads to nuclear accumulation of FOXO1, we used AS1842856, a selective small-molecule inhibitor of FOXO1 that directly binds nuclear-activated FOXO1 and prevents its interaction with target promoter sequences (48). This drug, therefore, allowed us to study whether FOXO1 inhibition could reverse the EHT 1610-induced $\mathrm{G}_{2}-\mathrm{M}$ delay. We found that FOXO1 inhibition alone induced a 2.5 -fold increase in $4 \mathrm{~N}$ cells in the late $\mathrm{G}_{2}-\mathrm{M}$ phase, as evidenced by CDK1 dephosphorylation (Figure 4, $\mathrm{E}$ and $\mathrm{F}$ ). We observed a similar increase in $\mathrm{G}_{2}-\mathrm{M}$ progression when AS1842856 was combined with EHT 1610. Furthermore, treatment of pre-B cells with AS1842856 partially rescued the proliferative defect induced by EHT 1610 (Figure 4G).

As mitigation of DNA damage is a major mechanism by which FOXO1 regulates $\mathrm{G}_{2}-\mathrm{M}$ progression, we assayed for $\gamma-\mathrm{H} 2 \mathrm{AX}$ foci in murine pre-B cells. We did not observe a significant increase in $\gamma$-H2AX foci with EHT 1610 treatment alone (Figure 4H). However, when EHT 1610 and AS1842856 were combined, we observed a cumulative increase in $\gamma$-H2AX foci. Notably, higher doses of AS1842856, alone or in combination with EHT 1610, markedly increased $\gamma$-H2AX foci, leading to high levels of cell death (Figure 4I). Thus, it is likely that loss of DYRK1A activity causes early cell-cycle progression, which is in turn negatively regulated by FOXO1 activation in order to ameliorate the resulting DNA damage from chromosomal instability. Indeed, RNA-Seq of Dyrk1adeficient small pre-B cells (17) revealed an upregulation of pathways involved in $\mathrm{G}_{2}-\mathrm{M}$ progression, DNA damage, the stress response, and, notably, FOXO signaling (Supplemental Figure 5, $\mathrm{B}$ and $\mathrm{C}$ ). This may be partly mediated by FOXO1 transcriptional activation of Gadd45a, which causes $\mathrm{G}_{2}-\mathrm{M}$ arrest in response to DNA damage $(45,49,50)$. Consistent with this hypothesis, we found that Gadd45a expression was increased upon EHT 1610 treatment but not when FOXO1 was inhibited alone or in combination with DYRK1A inhibition (Figure 4J).

FOXO1 regulation of the DNA damage response can be targeted in $B-A L L$. Although FOXO1 has been described as a tumor suppressor, it is overexpressed in B cell malignancies, including B-ALL, compared with its expression in other hematopoietic or solid tumors (Figure 5, A and B) (21). In our hands, FOXO1 protein levels were also increased in B-ALL cell lines and primary patient-derived cells compared with normal human BMMCs (Figure 5C). In order to assess how FOXO1 and DYRK1A cooperate in leukemogenesis, Nalm-6 and 697 cells, which respectively represent ETV6-PDGFRB and E2A-PBX subtypes of B-ALL, were treated with EHT 1610. Both B-ALL lines basally expressed high levels of nuclear FOXO1, which were increased further upon EHT 1610 treatment (Figure 5D). High basal levels of nuclear FOXO1 
A

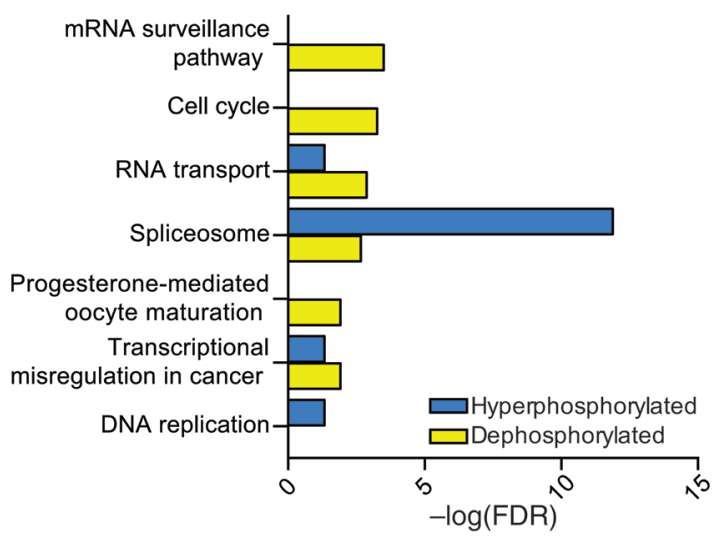

B

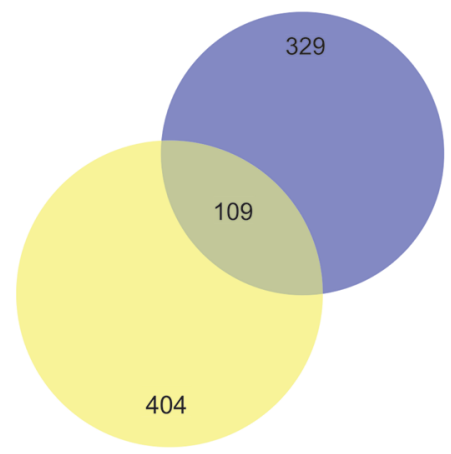

In vitro substrates

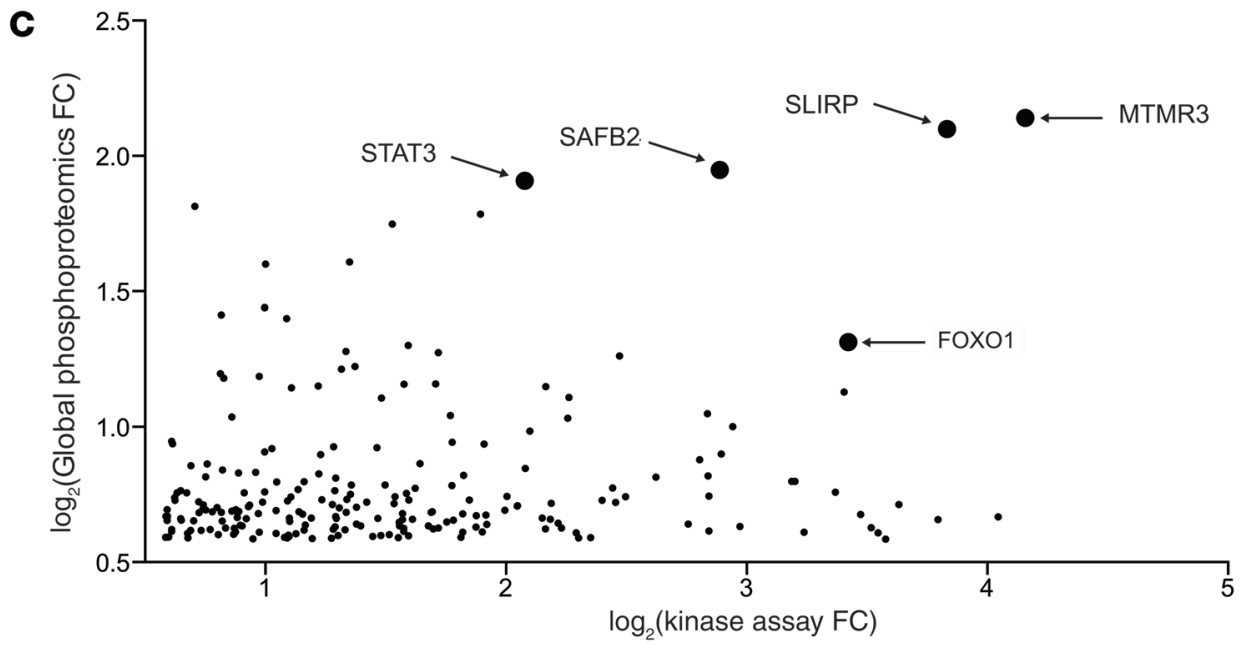

D

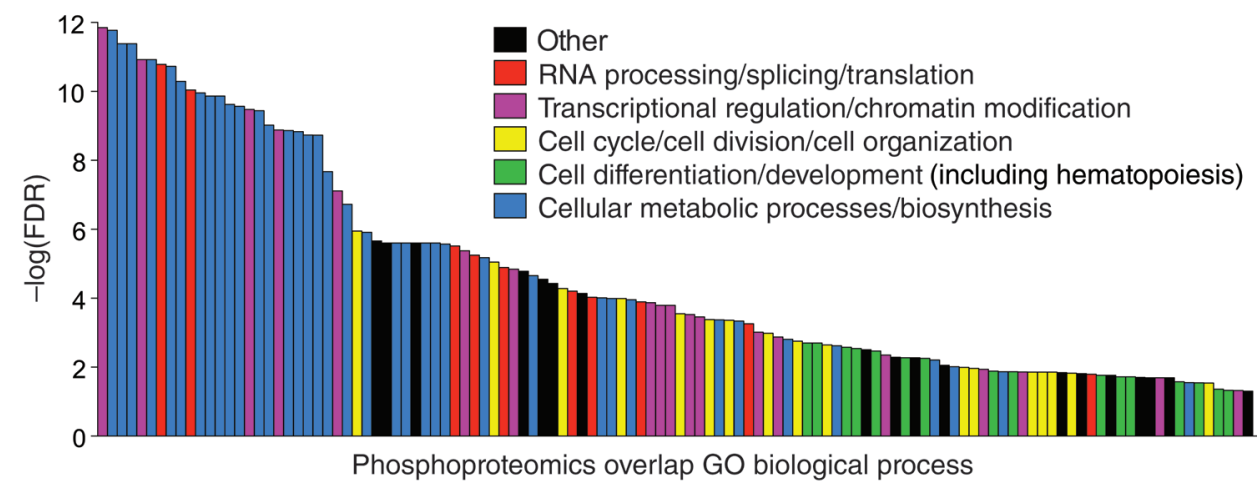

Figure 3. Kinase assay-linked phosphoproteomics identifies candidate DYRK1A substrates in pre-B cells. (A) Global differential phosphoproteomics analysis was performed on primary murine pre-B cells treated with $2 \mu \mathrm{M}$ EHT 1610 or DMSO for 2 hours. Peptides that were 1.5 -fold differentially phosphorylated were analyzed in the STRING database for upregulated KEGG (Kyoto Encyclopedia of Genes and Genomes) pathways. (B) Venn diagram showing overlapping peptides from the kinase-dependent phosphoproteome and in vitro substrates from the experiments described in Supplemental Figure 4, $A, E$, and $F$. Numbers indicate unique peptides in each cohort, and circle sizes indicate the relative size of the protein group. (C) Scatter plot depicting dephosphorylated peptides from global phosphoproteomics versus phosphorylated peptides from kinase assay phosphoproteomics. Arrows indicate the top 5 highest-scoring hits. FC, fold change versus the negative control. (D) A total of 109 overlapping peptides from $\mathbf{C}$ and $\mathbf{B}$ were analyzed in the STRING database for GO Biological Process enrichment. Individual annotations are functionally color-coded in the key. $n=2$ biological replicates for each proteomics assay $(\mathbf{A}-\mathbf{D})$. contrasted with our observations in WT murine pre-B cells. Moreover, higher FOXO1 expression was associated with poor EFS rates for patients with B-ALL (Supplemental Figure 6A), indicating that FOXO1 may not be a traditional tumor suppressor in B-ALL.

The high levels of nuclear FOXO1 in Nalm-6 and 697 cells suggest that it may be transcriptionally active and targetable in B-ALL, irrespective of the underlying genetics. Indeed, we observed that the leukemic samples were 3- to 200-fold more sensitive to AS1842856 than were primary murine pre-B cells (Figure 5E, Supplemental Figure 6B, and Supplemental Table 1), highlighting a substantial therapeutic window for FOXO1 inhibition. Notably, we treated an HeH-ALL cell line (MHH-CALL-2) as well as human PDX-passaged HeH-ALL cells (sample HeH-ALL-09) and DS-ALL cells (sample DS-ALL-02), all 3 of which contained increased DYRK1A copy numbers. We observed that these HSA21 aneuploid cells were among the B-ALL sample types that were most sensitive to FOXO1 inhibition. This enhanced sensitivity was also reflected in an independent assay of primary patient samples with increased DYRK1A copy numbers; 9 of 9 DS-ALL and $\mathrm{HeH}-$ ALL samples were sensitive to FOXO1 inhibition in the nanomolar range (Figure 5F and Supplemental Table 7).

Next, we investigated the mechanisms by which DYRK1A and FOXO1 inhibition led to impaired growth of leukemia cells. We noted that when Nalm-6 and 697 cells were treated with EHT 1610, there was minimal change in DNA damage compared with vehicle-treated cells (Figure 5G and Supplemental Figure 
A

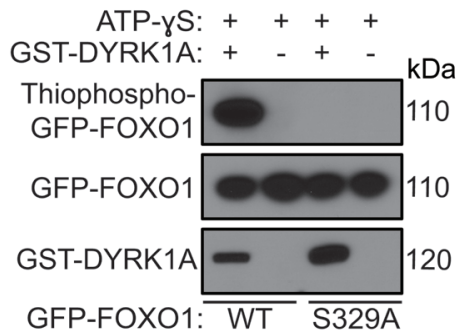

B

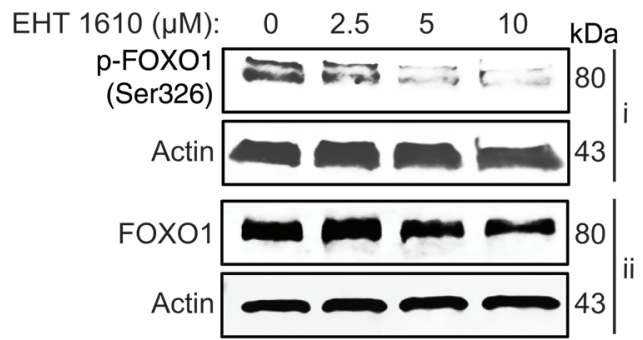

C

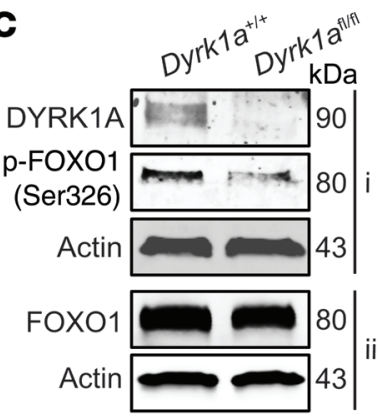

D

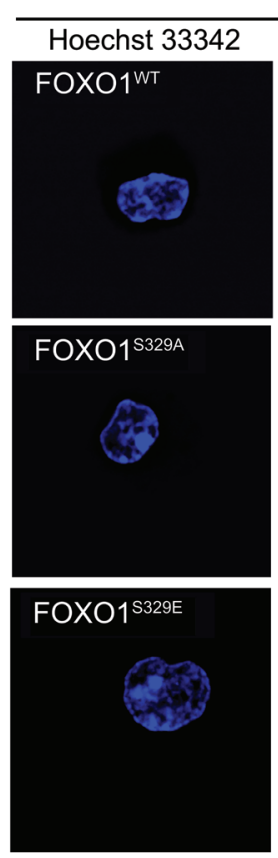

E

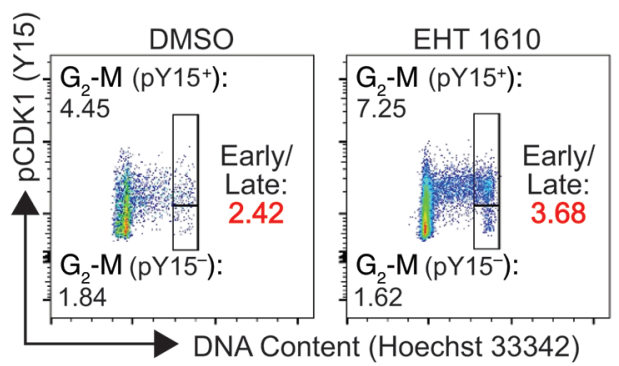

DMSO
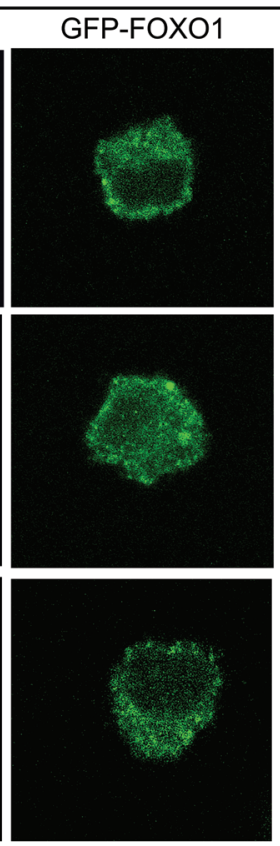
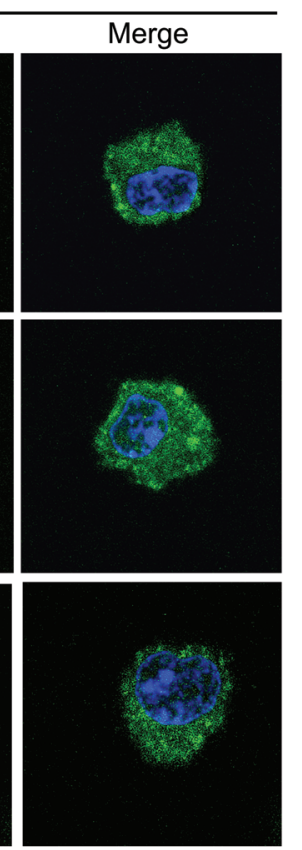
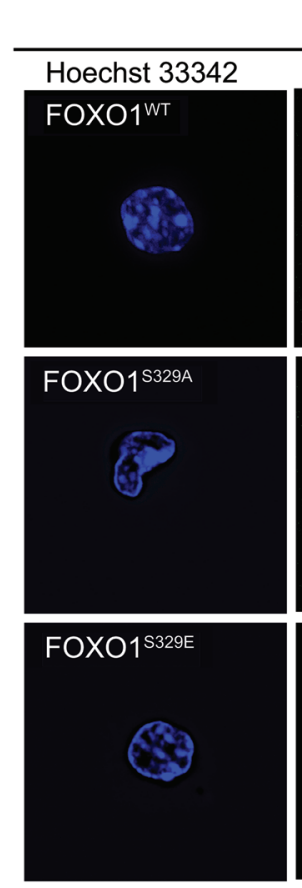

AS1842856
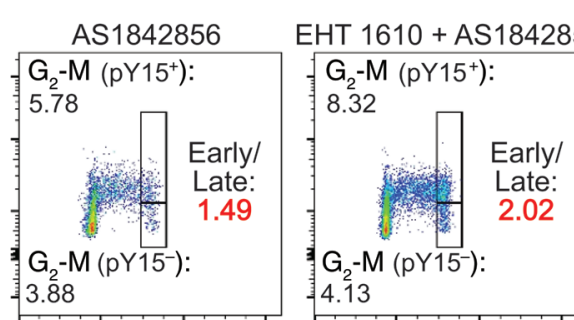

EHT 1610
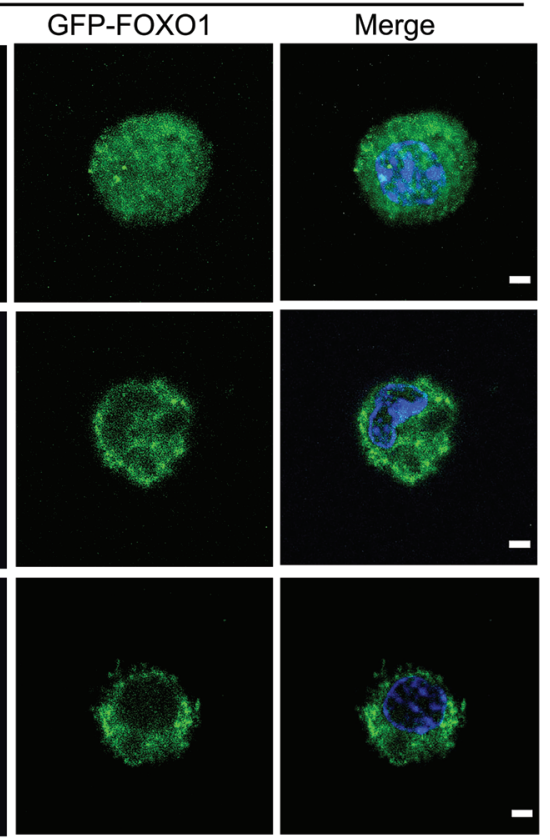

$\mathbf{F}$

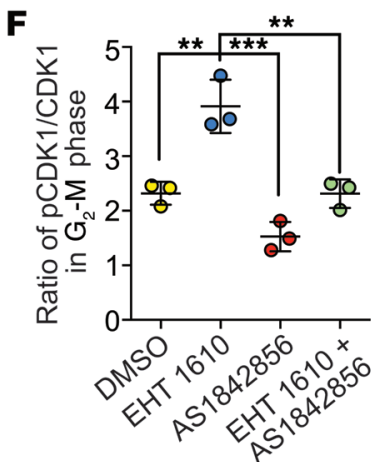

G

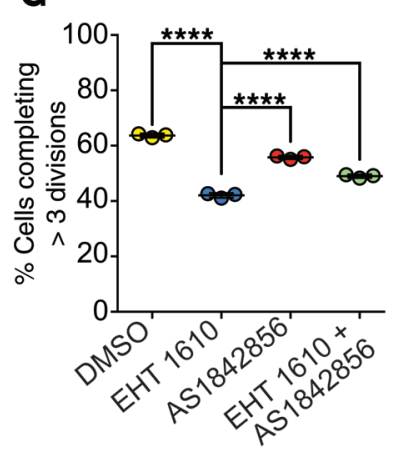

H

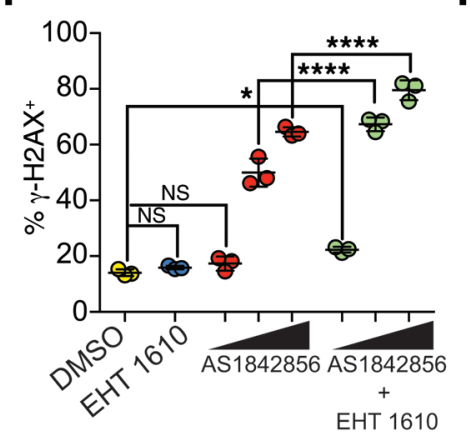

I

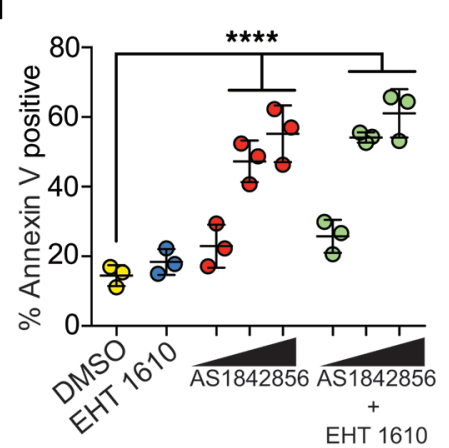

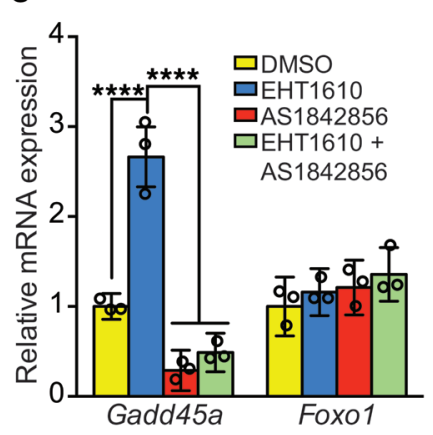


Figure 4. DYRIK1A phosphorylates FOX01 and regulates late cell-cycle progression. (A) Western blot results of an in vitro kinase assay with GST-DYRK1A, GFP-FOX01, and ATP- $\gamma$-S. Alkylated reaction products were analyzed for thiophosphate esters. (B) Western blot showing p-FOXO1 (Ser326) and total FOX01 protein in pre-B cells after 4 hours of treatment with EHT 1610 ( $i$ and ii indicate separate blots from the same cell extracts). (C) Western blot showing DYRK1A, p-FOXO1 (Ser326), and total FOXO1 protein in $D y r k 1 a^{+/+}$or Dyrk1a fl/fl pre-B cells after transduction with MIGR1-Cre ( $i$ and ii indicate separate blots from the same cell extracts). (D) GFP immunofluorescence of primary murine pre-B cells transfected with pcDNA-GFP-FOXO1 (WT, S329A, or S329E) for 48 hours. Cells were treated with DMSO or EHT 1610 for 4 hours and $10 \mu \mathrm{g} / \mathrm{mL}$ Hoechst 33342 for 1 hour prior to imaging. Images are representative of 4 independent experiments. Scale bars: $2.5 \mu \mathrm{m}$. (E) Representative flow cytometric plots of pre-B cell distribution in early $G_{2}-M\left(4 N, p Y 15^{+}\right)$or late $G_{2}-M(4 N$, pY15-) after a 48-hour treatment with EHT 1610, AS1842856, or both using p-CDK1 (Tyr15) versus DNA content. Numbers indicate the percentage of live cells in each gate. The early/late $\mathrm{G}_{2}-\mathrm{M}$ ratio is indicated in red. (F) Quantification of the early/late $G_{2}-M$ ratio based on gating in $\mathbf{E}$. Data indicate the mean \pm SD. (G) Quantification of CellTrace Violet dye dilution assay data after a 48-hour treatment with EHT 1610, AS1842856, or both. Data indicate the mean \pm SD. $(\mathbf{H})$ Quantification of the percentage of $\gamma-\mathrm{H}_{2} \mathrm{AX} \mathrm{X}^{+}$cells in primary murine pre-B cells treated with EHT 1610, AS1842856 (100 nM, $500 \mathrm{nM}$, or $1000 \mathrm{nM}$ represented by a scale bar), or both for 48 hours. Data indicate the mean \pm SD. (I) Quantification of flow cytometric analysis of apoptosis using annexin $\mathrm{V}$ staining in primary murine pre-B cells treated with EHT 1610, AS1842856 (100 nM, $500 \mathrm{nM}$, or $1000 \mathrm{nM}$ represented by a scale bar), or both for 48 hours. Data indicate the mean \pm SD. (J) Gadd45a and Foxo1 mRNA expression following qRT-PCR in primary murine pre-B cells after treatment with EHT 1610, AS1842856, or both. Data indicate the mean \pm SD (from triplicate wells of a representative sample). $n=3$ biological replicates (A-C and E-J). Significance was determined by ANOVA with post hoc Bonferroni's correction $(\mathbf{F}-\mathrm{J}) .{ }^{*} P<0.05,{ }^{* *} P<0.01,{ }^{* *} P<0.001$, and ${ }^{* * * *} P<0.0001$.

6C), although these cells still harbored at least a 2-fold increase in $\gamma$-H2AX foci at baseline compared with murine pre-B cells (compare Figure 5G with Figure 4H). When EHT 1610 was combined with $100 \mathrm{nM}$ AS1842856, we observed a marked increase in $\gamma$-H2AX foci compared with only a modest effect in WT pre-B cells at this same dose combination (Figure $4 \mathrm{H}$ ), indicating that leukemic cells have a greater dependence on FOXO1 regulation of DNA repair than do WT cells. Furthermore, expression of several FOXO1 target genes involved in DNA damage and the stress response, including GADD45A, CCNG2, and BCL2L11, was increased upon EHT 1610 treatment but was reduced when EHT 1610 and AS1842856 were combined, suggesting a possible sensitivity to DNA damage that can be exploited in leukemic cells (Figure 5H). Moreover, this combinatorial effect of EHT 1610 and AS1842856 on DNA damage in B-ALL cells caused a considerable decline in cell viability, underscoring the high degree of synergy when both targets were inhibited (Figure 5I, Supplemental Table 3, and Supplemental Table 4).

FOXO1 and DYRK1A are therapeutic targets in B-ALL. Given that EHT 1610 and AS1842856 suppressed the growth of B-ALL tumor cells in vitro, we next assayed whether these inhibitors recapitulated this activity in vivo. We first treated healthy C57BL/6 mice with EHT 1610 for 2 weeks to assess tolerance of the drug and on-target activity. Treatment with $40 \mathrm{mg} / \mathrm{kg} / \mathrm{day}$ EHT 1610 did not affect the peripheral WBC or platelet counts or hemoglobin levels but induced a modest decrease in the percentage of lym- phocytes (Supplemental Figure 7A). This peripheral blood phenotype was reflected in the bone marrow, with a significant reduction in $\mathrm{G}_{0}$-phase $\mathrm{CD} 19^{+}$cells (Supplemental Figure 7B). Furthermore, 3 days of treatment with $40 \mathrm{mg} / \mathrm{kg} /$ day EHT 1610 was associated with a loss of pre-B cell maturation at the large-to-small pre-B cell transition (Supplemental Figure $7 \mathrm{C}$ ) and a decrease in the degree of p-cyclin D3 in sorted pre-B cells (Supplemental Figure 7D). Together, these studies indicate that EHT 1610 is well tolerated and has the expected on-target activity. Next, we treated healthy C57BL $/ 6$ mice with $5 \mathrm{mg} / \mathrm{kg} /$ day and $10 \mathrm{mg} / \mathrm{kg} /$ day AS1842856 for 2 weeks. We did not observe any significant changes in peripheral blood counts at either dose level (Supplemental Figure 7E). However, upon bone marrow analysis, we noted a dose-dependent reduction in pre- $\mathrm{B}$ cells, consistent with the documented role of FOXO1 in B lymphopoiesis $(44,49)$ (Supplemental Figure 7F).

As EHT 1610 and AS1842856 are well tolerated and have ontarget effects in vivo, we transplanted NOD.Cg-Prkdcsid Il2rg $g^{\text {tmIWjl } /}$ SzJ (NSG) mice with human B-ALL cells to determine whether they could be treated effectively. We transplanted MHH-CALL-4 cells into 1 cohort of NSG mice and treated them with $40 \mathrm{mg} / \mathrm{kg}$ / day EHT 1610, and then transplanted Nalm-6 cells into a separate cohort of NSG mice followed by treatment with $10 \mathrm{mg} / \mathrm{kg} / \mathrm{day}$ AS1842856. Note that the 2 different lines were used on the basis of our determined sensitivities (Supplemental Table 1). In both cases, the mice were treated for 2 weeks following detection of $1 \%$ or more human $\mathrm{CD} 45^{+} \mathrm{CD} 19^{+}$cells in the peripheral blood. Both cohorts displayed highly significant survival advantages (Figure 6, A and B).

We also evaluated the degree of antileukemia activity in 2 PDX models of HSA21 aneuploidy (22). First, we transplanted NSG mice with luciferase-expressing leukemic blasts from a patient with DS-ALL (sample DS-ALL-03) to follow leukemia progression by noninvasive in vivo imaging. When the recipient mice reached $10^{7}$ photons $/$ second $(\mathrm{p} / \mathrm{s})$ in total flux, we treated the animals for 3 weeks with $40 \mathrm{mg} / \mathrm{kg} /$ day EHT 1610 or $30 \mathrm{mg} /$ $\mathrm{kg} /$ day AS1842856. EHT 1610 treatment alone conferred a 20\% decrease in leukemic burden compared with the control group versus a more than 100-fold decrease in the AS1842856 group as determined by bioluminescence (Figure 6C). These reductions in leukemic burden correlated with significant survival advantages compared with vehicle treatment, with AS1842856 providing a greater survival benefit than EHT 1610 (Figure 6D).

Last, we also evaluated the efficacy of EHT 1610 and AS1842856 in a highly aggressive luciferase-expressing PDX model of HeH-ALL (sample HeH-ALL-09). After detection of $10^{7}$ $\mathrm{p} / \mathrm{s}$ in total flux, we treated transplanted mice for 3 weeks with 40 mg/kg/day EHT 1610 or 30 mg/kg/day AS1842856. EHT 1610 treatment in this aggressive model reduced leukemic burden by approximately $8 \%$ and conferred a modest survival advantage (Figure 6, E and F); by contrast, AS1842856 treatment reduced the leukemic burden by approximately 10 -fold at endpoint analysis and provided an even stronger survival benefit. Thus, these data demonstrate that DYRK1A and FOXO1 are both efficacious targets in B-ALL and may be of particular therapeutic value in models of disease with chromosome 21 aneuploidy.

DYRK1A regulates B-ALL cell growth through phosphorylation of STAT3. Although targeting the DYRK1A/FOXO1 axis demonstrates therapeutic efficacy in a variety of B-ALL subtypes through 
A

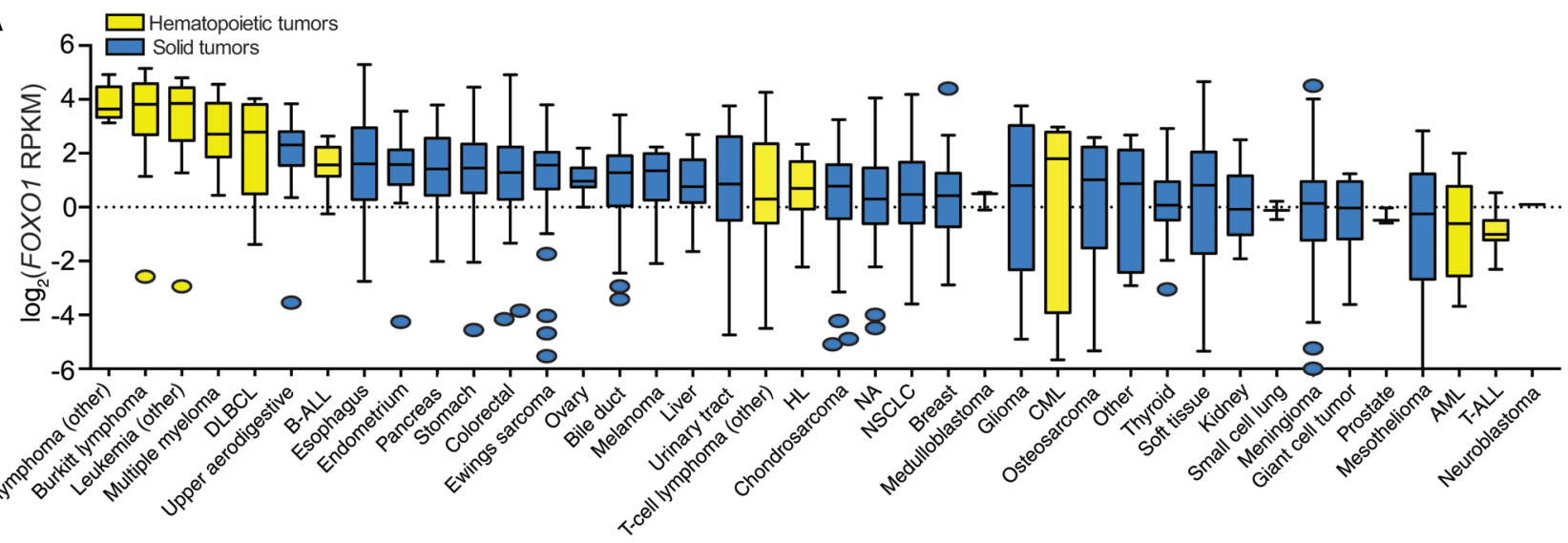

B

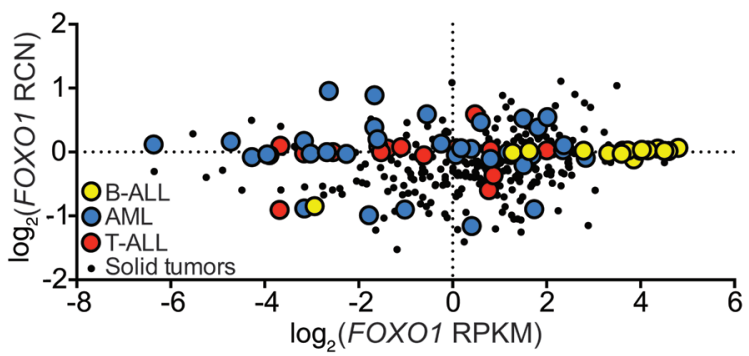

D

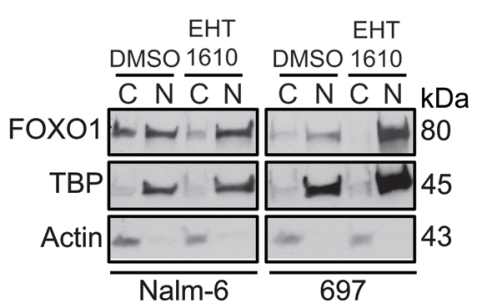

H
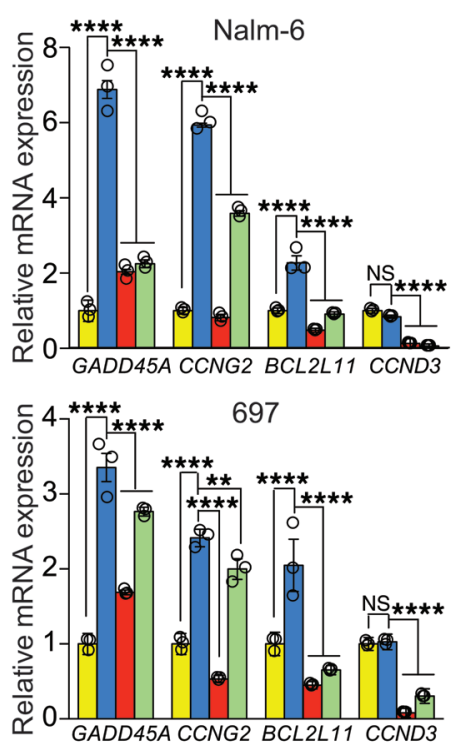

E
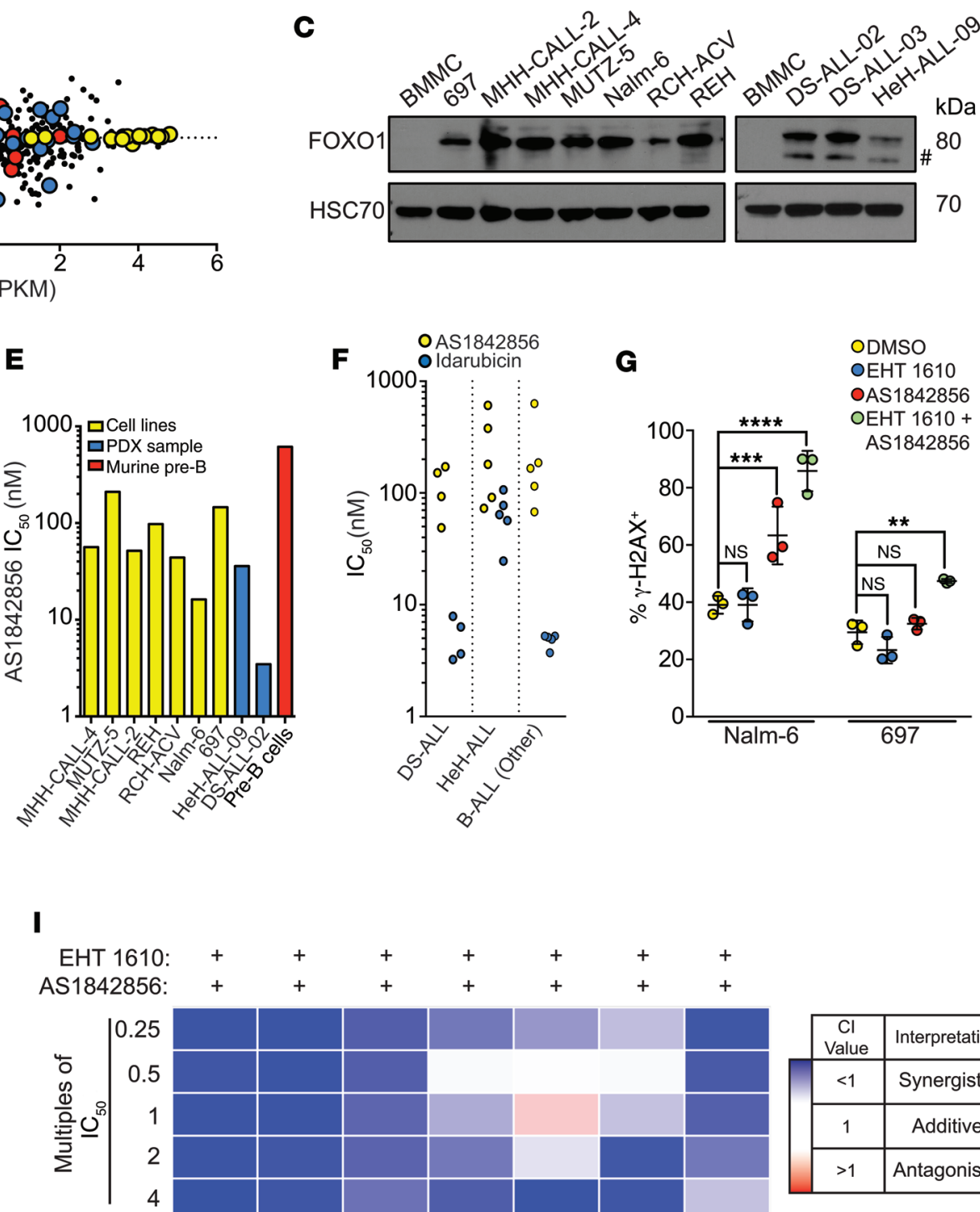

$\square$ DMSO $\square$ AS1842856

$\square$ EHT $1610 \square$ EHT $1610+$ 
Figure 5. FOX01 is required for the B-ALL DNA damage response and can be targeted with AS1842856. (A) FOXO1 mRNA expression (RPKM) distribution across cell lines from the Broad Institute's CCLE, ordered by median FOXO1 expression levels (line), the IQR (box), and up to 1.5 times the IQR (bars). HL, Hodgkin lymphoma. (B) FOXO1 mRNA expression versus the RCN across B-ALL $(n=16)$, T-ALL $(n=16), \operatorname{AML}(n=35)$, and solid tumors (non-small cell $[n=122]$ and small cell $[n=50]$ lung, colorectal [ $n$ $=57]$, breast $[n=57]$, and prostate $[n=8]$ ) from the CCLE. Dots represent individual cell lines. (C) Western blot showing total FOXO1 protein in B-ALL cell lines (MHH-CALL-4, MUTZ-5, MHH-CALL-2, REH, RCH-ACV, 697, and Nalm-6), patient samples (DS-ALL-02, DS-ALL-03, and HeH-ALL-09), and human BMMCs. Data are from the same gel as in Figure $1 \mathrm{C}$, which was separately probed for DYRK1A. Pound sign indicates the nonspecific band. (D) Western blot showing total FOX01 protein in Nalm-6 and 697 cells after treatment with EHT 1610 for 8 hours. C, cytoplasmic; N, nuclear. (E) $\mathrm{IC}_{50}$ values for B-ALL cell lines, patient samples, and primary murine pre-B cells treated with AS1842856 for 48 hours, based on data in Supplemental Figure 6B. (F) $I C_{50}$ values for patient samples (DS-ALL, HeH-ALL, and nonaneuploid B-ALL control) treated with AS1842856 or idarubicin. Dots represent 3 biological replicates of different patient samples. (G) Quantification of the percentage of $\gamma-\mathrm{H}_{2} \mathrm{AX}{ }^{+}$cells from the data in Supplemental Figure 6B. Data indicate the mean \pm SD. (H) FOX01 target gene mRNA expression from qRT-PCR in Nalm- 6 (top) and 697 (bottom) cells after treatment with EHT 1610, AS1842856, or both. Data indicate the mean \pm SD (from triplicate wells of a representative sample). (I) Heatmap of CI values when combining EHT 1610 with AS1842856 at the multiples of $I_{50}$ values indicated in Supplemental Table 3. $n=3$ biological replicates $(\mathbf{C}-\mathbf{I})$. Significance was determined by ANOVA with post hoc Bonferroni's correction (G and $\mathbf{H}$ ). ${ }^{* *} P<0.01,{ }^{* * *} P<0.001$, and ${ }^{* * * *} P<0.0001$.

DNA damage regulation, this mechanism does not entirely explain why leukemic cells with JAK pathway hyperactivation (Ph-like ALL [MUTZ-5, MHH-CALL-4] and DS-ALL [DS-ALL-02]) were exquisitely sensitive to EHT 1610 monotherapy. Thus, we were prompted to investigate the role of other candidate DYRK1A substrates in B-ALL. One of the highest-scoring hits in our global phosphoproteomics assay was the Ser727 residue of STAT3. Therefore, we first validated that DYRK1A phosphorylates the Ser727 residue of STAT3 using an in vitro kinase assay (Figure 7A). This phosphorylation event was diminished in MUTZ-5 cells with both EHT 1610 treatment and targeting of DYRK1A (Figure 7, B and C). To determine whether the efficacy of DYRK1A inhibition in MUTZ-5 cells was primarily mediated through STAT3 activity, we overexpressed WT, phosphomimetic (S727D, S727E), and phosphodeficient (S727A) alleles of STAT3 in these cells and found that expression of the phosphomimetic alleles significantly reduced their sensitivity to EHT 1610 (Figure 7D). Conversely, expression of the phosphodeficient allele yielded an $\mathrm{IC}_{50}$ similar to that of the empty vector, emphasizing the requirement of Ser727 for EHT 1610 sensitivity. Moreover, we observed an increase in apoptosis of MUTZ-5 cells upon EHT 1610 treatment, which was then reduced by overexpression of phosphomimetic alleles of STAT3 (Figure 7E), thus suggesting that STAT3 Ser727 phosphorylation by DYRK1A is critical for Ph-like ALL survival.

The precise role of STAT3 Ser727 phosphorylation remains unclear, with previous reports implicating it in canonical JAK/ STAT signaling $(41,51,52)$, noncanonical transcriptional regulation $(53,54)$, and regulation of the mitochondrial electron transport chain (55-57) and mitochondrial ROS (55-60). In support of the idea of an effect on JAK/STAT signaling, we observed that loss of DYRK1A activity was not only associated with decreased Ser727 phosphorylation, but Tyr705 phosphorylation as well, suggesting that Ser727 phosphorylation may impact JAK-mediated phosphorylation of Tyr705, possibly by acting as a priming event (Figure 7, B and C). Expression of phosphomimetic (S727D, $\mathrm{S} 727 \mathrm{E}$ ) alleles was also associated with a significant reduction in mitochondrial ROS in the setting of EHT 1610 treatment compared with that seen with WT and phosphodeficient alleles (Figure 7F). These findings suggest that STAT3 Ser727 phosphorylation may be protective in leukemic cells by ameliorating the production of mitochondrial ROS.

Clinically, high expression of STAT3 is associated with worse EFS rates, although this appears to be more prominent in subtypes with altered JAK/STAT signaling, namely Ph-like ALL (Supplemental Figure 8). Therefore, we treated MHH-CALL-4 and MUTZ-5 cells with C188-9, a STAT3-selective small-molecule inhibitor that is currently in clinical trials for several common solid tumors including head and neck squamous cell carcinoma $(61,62)$ and non-small cell lung cancer (NSCLC) (63). Both Ph-like ALL cell lines were sensitive to C188-9, but Nalm-6 cells, which lack activation of the JAK/STAT pathway, were insensitive to STAT3 inhibition (Figure 7G). Similar sensitivity to C188-9 was seen in human PDX-passaged Ph-like ALL samples, with driving lesions affecting JAK/STAT signaling (Figure $7 \mathrm{H}$ and Supplemental Table 8). Of note, improved STAT3 inhibitors, such as the recently described STAT3 PROTAC may show profoundly better activity in vivo and vitro $(64,65)$. These data indicate that in B-ALL with enhanced JAK signaling, selective inhibition of STAT3 represents a potential therapeutic approach.

\section{Discussion}

B-ALL patients with DS have high rates of treatment-related morbidities, such as methotrexate-related mucositis $(66,67)$ and corticosteroid-related hyperglycemia (67), which may increase mortality and thus limit dose escalation, ultimately leading to higher relapse rates and a worse outcome, with a 5-year EFS rate of 55\%$70 \%$ (5). Here, we reveal that DYRK1A is a therapeutic target in DS-ALL as well as in other B-ALL subtypes with HSA21 aneuploidy. We discovered that inhibition of DYRK1A sensitized B-ALL blasts to cytotoxic chemotherapy by induction of cycling, similar to the concept of granulocyte CSF (G-CSF) priming in AML (68). Targeting DYRK1A is of significant interest to the medical community, as studies have suggested that its increased activity is responsible for numerous neuronal sequelae of DS. For example, green tea polyphenols, such as epigallocatechin gallate (EGCG), have been tested in mouse models of DS and found to increase cognition $(69,70)$. Of note, green tea polyphenols and the other commonly used DYRK1A inhibitor harmine target a wide range of kinases; thus, the role of DYRK1A in these pathologies remains incompletely defined. By contrast, we leveraged the most highly selective and potent DYRK1A inhibitor EHT 1610 (28-31) to show that DYRK1A is indeed a therapeutic target in B-ALL. This conclusion is supported by genetic data that showed that even heterozygous loss of Dyrk1a almost completely ablated the leukemia-initiating activity of the p190 isoform of BCR-ABL. Although BCR-ABL expression is uncommon in DS-ALL, the most common chromosomal aneuploidy in BCRABL-positive ALL is polysomy 21 (71). 
A
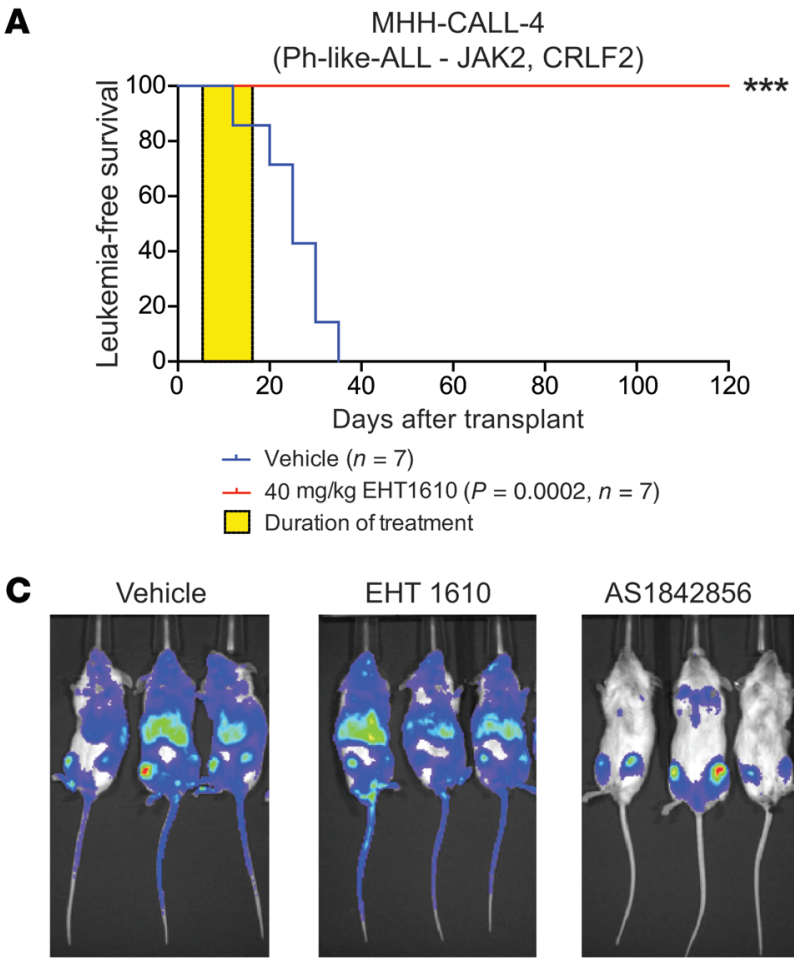

$\times 10^{9}$

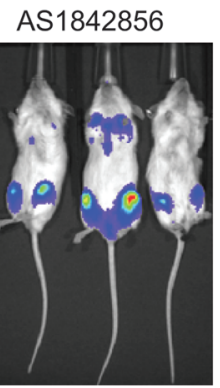

$\times 10^{9}$

$0.2 \quad 0.4 \quad 0.6 \quad 0.8$

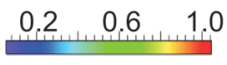

Radiance $\left(\mathrm{p} / \mathrm{s} / \mathrm{cm}^{2} / \mathrm{sr}\right)$

E

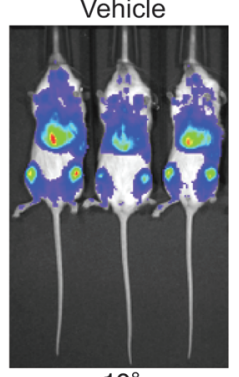

$\times 10^{8}$

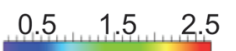

Radiance $\left(\mathrm{p} / \mathrm{s} / \mathrm{cm}^{2} / \mathrm{sr}\right)$

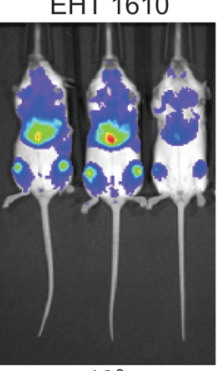

$\times 10^{8}$

0.51 .01 .52 .0

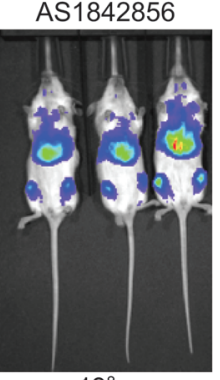

$\times 10^{8}$

$\begin{array}{lll}0.2 & 0.6 & 1.0\end{array}$ r)
B

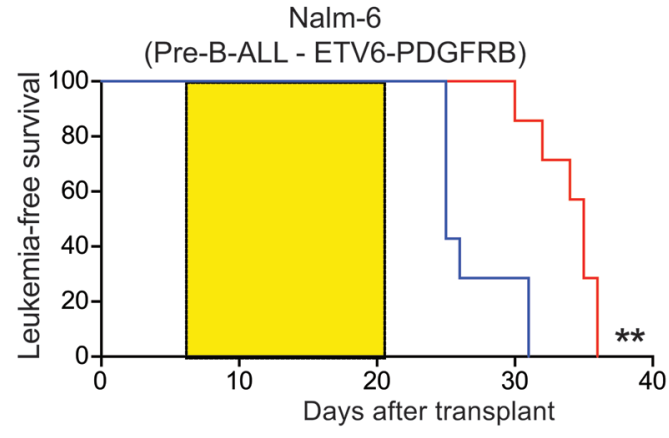

- Vehicle $(n=7)$

$+10 \mathrm{mg} / \mathrm{kg} \mathrm{AS} 1842856(P=0.001, n=7)$

Duration of treatment

D

DS-ALL-03

(DS-ALL - CRLF2, PAX5, CDKN2A, KDM6A)

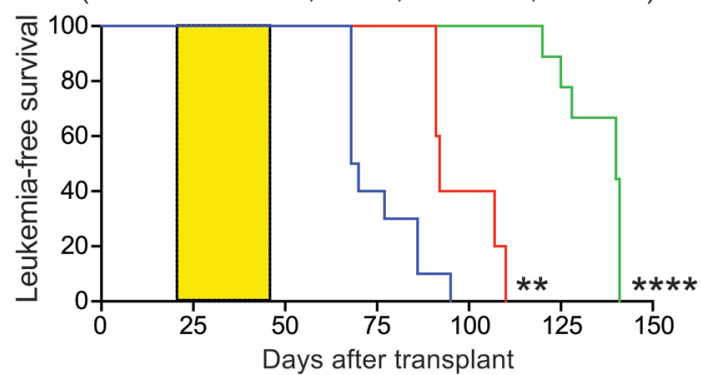

$+\operatorname{Vehicle}(n=9)$

- $40 \mathrm{mg} / \mathrm{kg} \mathrm{EHT1610}(P=0.008, n=5)$

$+30 \mathrm{mg} / \mathrm{kg}$ AS1842856 $(P<0.0001, n=9)$

Duration of treatment

F
HeH-ALL-09

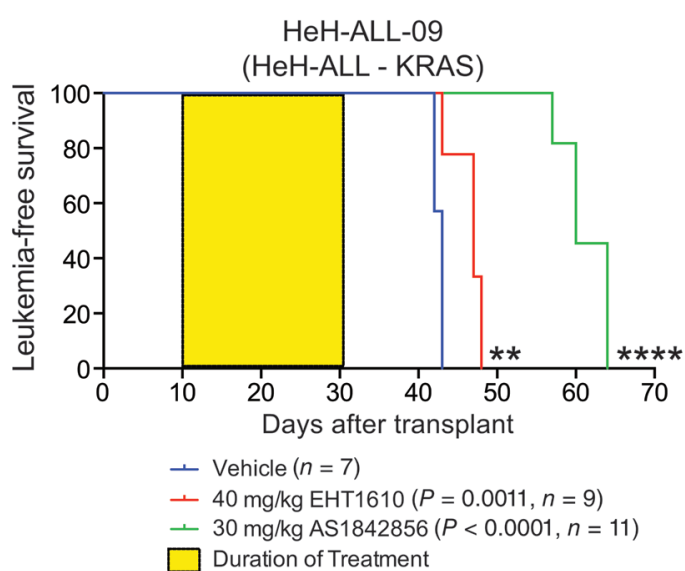

Figure 6. FOXO1 and DYRK1A are therapeutic targets in B-ALL. (A and B) MHH-CALL-4 and Nalm- 6 cell lines were transplanted into NSC mice, and the animals were treated with EHT 1610 or AS1842856, respectively, for 2 weeks (yellow boxes). Kaplan-Meier analysis of mice treated with EHT 1610 (A) or AS1842856 (B) was performed. Graph titles describe the genetic backgrounds of the transplanted leukemia cells. $P$ values and sample sizes ( $n$ ) are shown in the key. (C) NSC mice were transplanted with a DS-ALL-03 luciferase-expressing PDX sample and treated with EHT 1610 or AS1842856. Representative images of leukemic burden within each treatment cohort are shown. Radiance scales are shown below each image. (D) Kaplan-Meier analysis of DS-ALL-03 cohorts after 3 weeks (yellow box) of treatment. Graph title describes the genetic background of the leukemia. $P$ values and sample sizes $(n)$ are shown in the key. (E) NSG mice were transplanted with the HeH-ALL-09 luciferase-expressing PDX sample and treated with EHT 1610 or AS1842856. Representative images of leukemic burden within each treatment cohort at the treatment endpoint are shown. Radiance scales are shown below each image. (F) Kaplan-Meier analysis of HeH-ALL-09 cohorts after 3 weeks (yellow box) of treatment. Graph title describes the genetic background of the leukemia. $P$ values and sample sizes ( $n$ ) are shown in the key. Significance was determined by log-rank (Mantel-Cox) test (A, B, D, and F). ${ }^{* *} P<0.01{ }^{* * *} P<0.001$, and ${ }^{* * *} P<0.0001$. sr, steradian.

Mechanistically, we performed an unbiased substrate analysis of DYRK1A in hematopoietic cells and revealed that DYRK1A phosphorylation of FOXO1 is critical for B cell development through late cell-cycle regulation in response to DNA dam- age. While the precise interplay between DYRK1A- and PI3K/ AKT-mediated regulation of FOXO1 intracellular localization remains unclear, it is possible that Ser326/Ser329 phosphorylation facilitates FOXO1 nuclear export, since this motif lies 
A
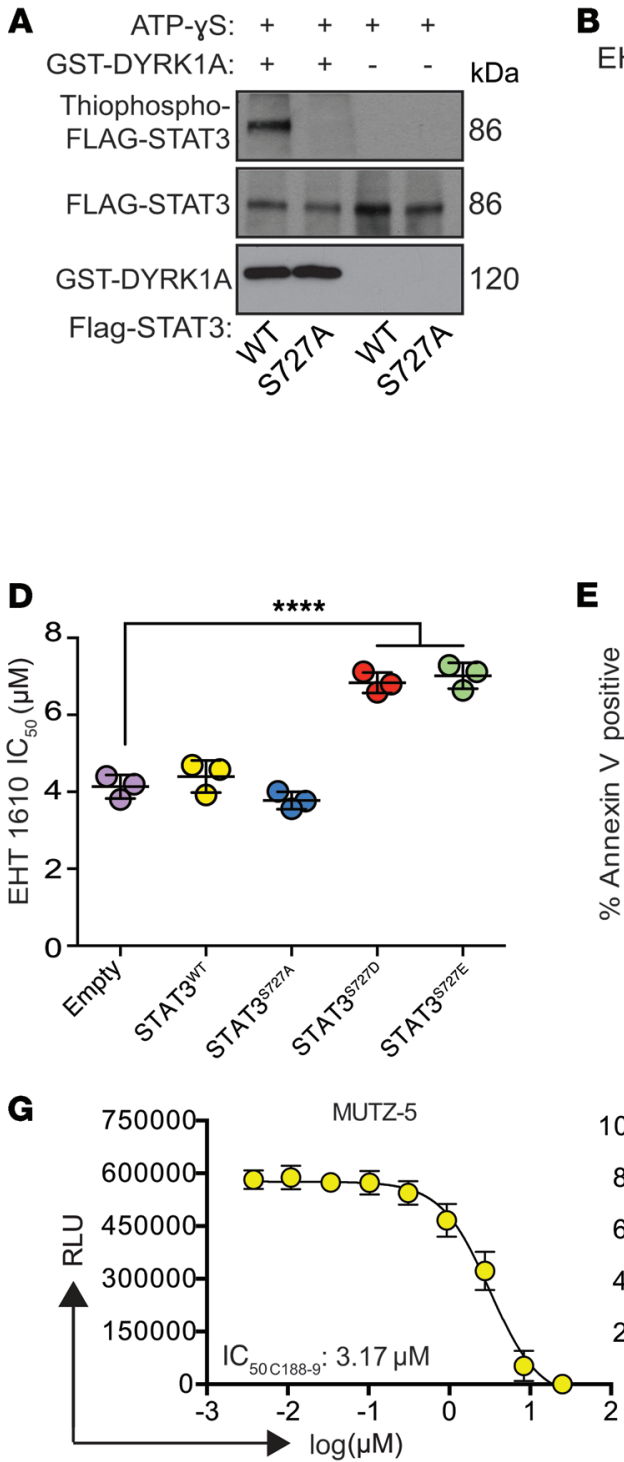

H

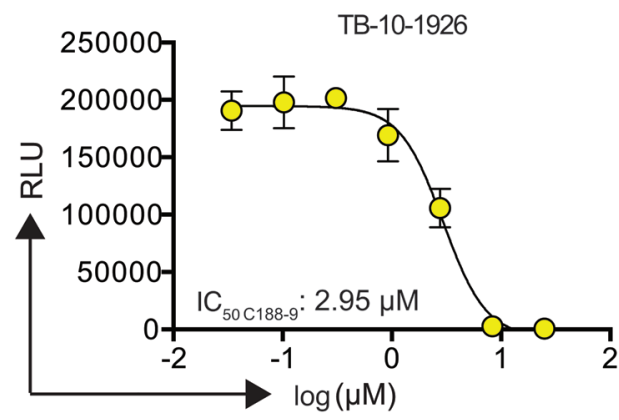

B

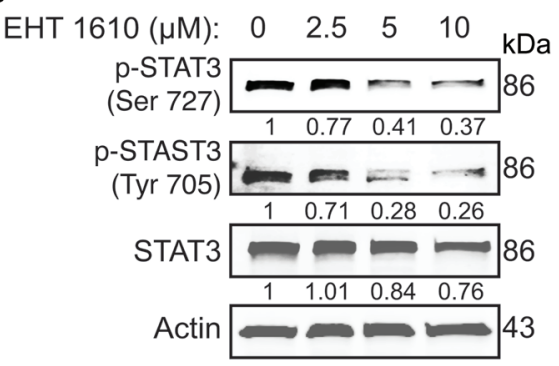

C
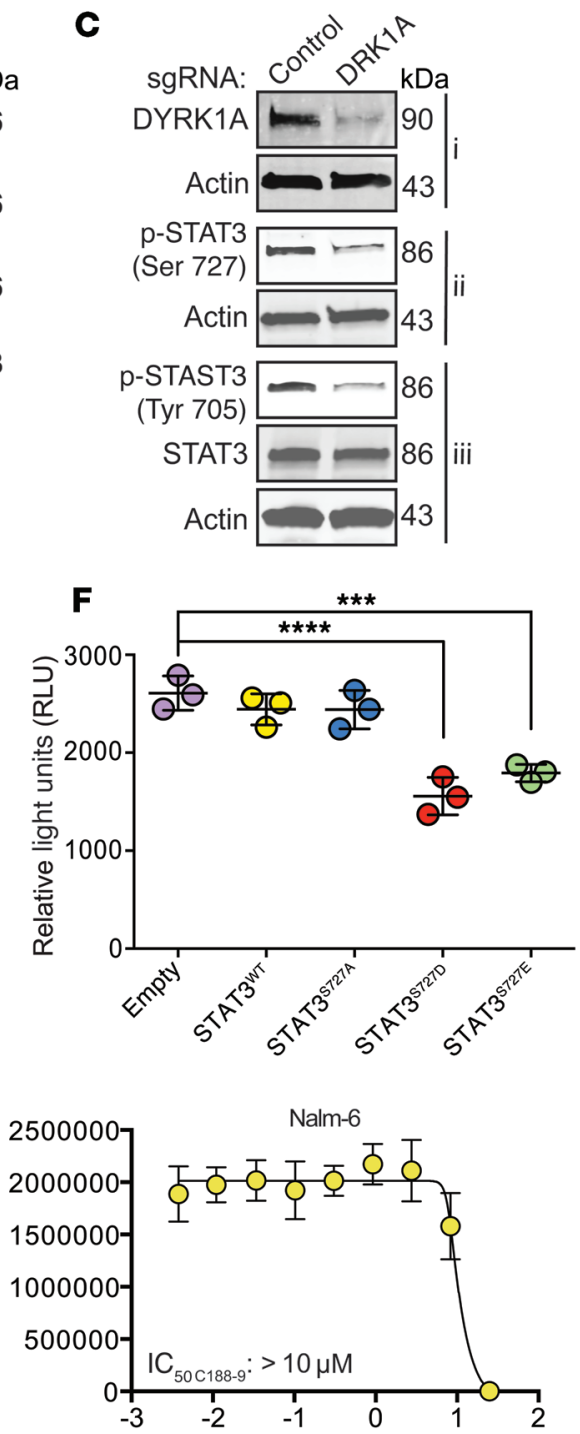

E

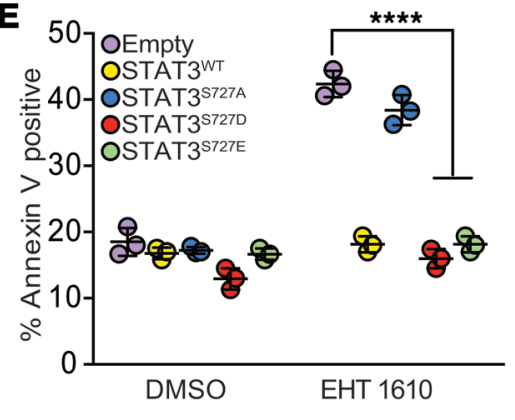

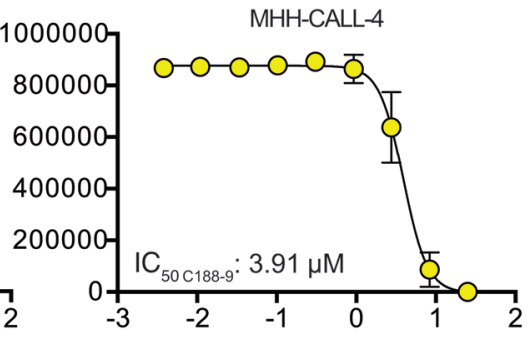

PANNGL

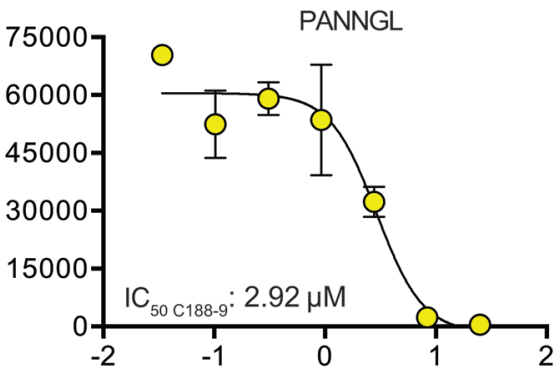

Figure 7. DYRK1A-mediated phosphorylation of STAT3 regulates mitochondrial ROS in B-ALL. (A) Western blot results of an in vitro kinase assay with GST-DYRK1A, FLAG-STAT3, and ATP- $\gamma$-S. Alkylated reaction products were analyzed for thiophosphate esters. (B) Western blot showing p-STAT3 (Ser727, Tyr705) and total STAT3 protein in MUTZ-5 cells after 4 hours of treatment with EHT 1610. Densitometric values were normalized to actin. (C) Western blot showing p-STAT3 (Ser727, Tyr705), total STAT3, and DYRK1A in MUTZ-5 cells with or without CRISPR/Cas9-mediated knockout of DYRK1A (i, ii, and iii indicate separate blots from the same cell extracts). (D) IC Fo $_{50}$ values of MUTZ-5 cells transduced with MSCV-puro (empty, STAT3 ${ }^{\text {WT }}$, STAT3 ${ }^{\text {S727A }}$, STAT3 ${ }^{\text {S727D, }}$, or STAT3 ${ }^{\text {S727E) }}$ after treatment with $3 \mu \mathrm{M} \mathrm{EHT} \mathrm{1610.} \mathrm{Data} \mathrm{indicate} \mathrm{the} \mathrm{mean} \pm$ SD. (E) Quantification of flow cytometric analysis of apoptosis using annexin $V$ staining in MUTZ-5 cells transduced with MSCV-puro (empty, STAT3 ${ }^{\text {WT }}$, STAT3 ${ }^{\text {S727A }}$, STAT3 ${ }^{\text {S7270 }}$, or STAT3 ${ }^{\text {S727E) after treatment with } 3 \mu M}$ EHT 1610 . Data indicate the mean \pm SD. (F) Mitochondrial ROS luminescence quantification as RLU (relative light units) after treatment with $3 \mu \mathrm{M}$ EHT 1610 using the

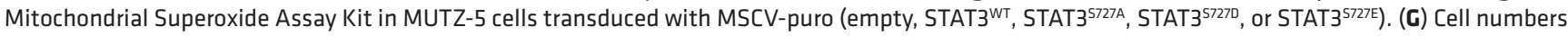
after treatment of B-ALL cell lines with C188-9, quantified by luminescence as RLU. Curves were fitted nonlinearly with variable slope (4 parameters). IC ${ }_{50}$ values are shown. (H) Cell numbers after treatment of human PDX-passaged Ph-like ALL samples with C188-9, quantified by luminescence as RLU. Curves were fitted nonlinearly with variable slope (4 parameters). $I C_{50}$ values are shown. $n=3$ biological replicates (A-H). Significance was determined by ANOVA with post hoc Bonferroni's correction (D-F). ${ }^{* *} P<0.001$ and ${ }^{* * *} P<0.0001$. 
adjacent to the FOXO1 nuclear export sequence (72). This is a mechanism similar to the well-known role of DYRK1A in regulating NFAT nuclear export (73). Thus, upon DYRK1A inhibition, FOXO1 nuclear export would be delayed or abrogated, allowing for subsequent transcription of late cell-cycle regulatory genes such as Gadd45a. Notably, upregulation of Gadd45a downstream of FOXO1 transactivation has been reported to be critical for transcription of the Rag1-Rag2 locus in B cell development (49) and may, therefore, explain why Rag1 transcripts are appropriately increased in Dyrk1a-deficient pre-B cells despite the loss of other pre-BCR signaling components (17). Moreover, we previously observed that loss of DYRK1A does not increase apoptosis in pre-B cells despite this $\mathrm{G}_{2}-\mathrm{M}$ arrest; although FOXO1 is known to induce apoptosis, it is unclear whether this mechanism occurs in a cell-cycle-dependent manner or whether $\mathrm{G}_{2}-\mathrm{M}$-arrested cells are specifically resistant to FOXO1-mediated apoptosis (45).

DYRK1A is a known cell-cycle regulator with several substrates, including Lin52 (15), P27(Kip1) (16), and cyclin D1 (18); however, our findings reveal that DYRK1A controls different stages of the cell cycle through nonredundant mechanisms: it negatively regulates $\mathrm{G}_{1}-\mathrm{S}$ progression by phosphorylation of cyclin D3 (17) and positively regulates $\mathrm{G}_{2}-\mathrm{M}$ progression via FOXO1 phosphorylation. These effects underscore the ongoing question of whether DYRK1A acts as a tumor suppressor or an oncogene, as there is conflicting evidence for its role as either in the traditional sense. For example, in glioblastoma multiforme, Pozo et al. observed that DYRK1A inhibition reduced cell survival by destabilization of the EGFR (74). By contrast, Lee et al. reported that DYRK1A phosphorylation of ID2 reduced tumor burden through HIF $2 \alpha$ destabilization (75).

Although FOXO1 canonically acts as a tumor suppressor by negatively regulating $\mathrm{G}_{1}-\mathrm{S}$ and $\mathrm{G}_{2}-\mathrm{M}$ progression, it is potently targetable in B-ALL. Although the presence of high nuclear FOXO1 levels in B-ALL is paradoxical to its typical role as a tumor suppressor, its oncogenic role is not unprecedented, particularly in hematopoietic malignancies, as FOXO1-activating mutations have been associated with poor prognosis in diffuse large B cell lymphoma (DLBCL) (76). In Burkitt lymphoma, nuclear FOXO1 has specifically been found to promote tumorigenesis (77). Additionally, Skyes et al. illustrated that FOXO1 is involved in the maintenance of the leukemia-initiating cell state in AML (78). Our work also independently validates the findings by Wang et al. in B-ALL (79); however, we have expanded this to find that FOXO1 was selectively targetable in ALL with HSA21 aneuploidy through exploitation of FOXO1-mediated DNA damage. Moreover, while CCND3 has been proposed as a FOXO1 target involved in B-ALL (79), its expression is not significantly altered with EHT 1610 treatment, consistent with our prior observations in Dyrk1a-deficient pre-B cells (17). This suggests that DYRK1A regulation of cyclin D3 stability at the protein level, thereby leading to negative feedback of its expression, may be a more dominant phenotype than FOXO1mediated changes in CCND3 expression downstream of DYRK1A inhibition. Thus, it is possible that, although DYRK1A and FOXO1 have documented pro- and antitumorigenic roles, these may be context dependent in normal versus malignant hematopoiesis, and manipulation of these critical factors that control self-renewal in leukemia may be a powerful multifocal therapeutic approach.
We also identified STAT3 as a substrate of DYRK1A that contributes to the maintenance of B-ALL cells with JAK activation, underscoring a multifactorial role for DYRK1A in oncogenesis. STAT3 Ser727 has previously been reported as a DYRK1A substrate in murine DS models of astrogliogenesis (41); however, this interaction has not been previously demonstrated in hematopoietic cells. Our data suggest that phosphorylation of Ser727 has at least 2 effects. First, we observed a striking reduction in the degree of Tyr705 phosphorylation with DYRK1A loss, indicating that JAK/STAT signaling is likely enhanced by DYRK1A under normal conditions. Second, phosphorylation of STAT3 at Ser727 has been reported to mediate its mitochondrial localization and subsequent electron transport chain regulation $(56,60)$. Of note, we detected a reduction in the level of mitochondrial ROS in cells that express phosphomimetic alleles of Ser727, consistent with an effect of DYRK1A phosphorylation on the mitochondrial function of STAT3. Although we have not established whether canonical nuclear or noncanonical mitochondrial STAT3 activity mediates its contribution to B-ALL, our data suggest that STAT3 may be a therapeutic target, particularly in Ph-like ALL. Indeed, Ph-like ALL often contains CRLF2 overexpression and/or JAK2-activating mutations, both of which increase JAK/STAT signaling (80); this subtype of ALL has a poor prognosis, with adult patients having a 5-year EFS rate of 24\%-58\% (81). Tyrosine kinase inhibitors such as ruxolitinib are currently under study in Ph-like ALL (ClinicalTrials.gov NCT03571321); however, one of the major pitfalls of ATP-mimetic tyrosine kinase inhibitors is the eventual development of resistance (82). Thus, targeting downstream signaling components, like STAT3, is a potential avenue by which to overcome this barrier.

There have been major improvements in the treatment of B-ALL over the past few decades, including potent multidrug regimens and the development of anti-CD19 therapy via CAR-T cells or bispecific antibodies; however, these therapies have significant adverse effects, particularly in patients with DS, and are often not curative. Therefore, the development of novel targeted therapies is still critical for improving the care of patients with B-ALL.

\section{Methods}

Additional details can be found in the Supplemental Methods.

Animal studies. $\mathrm{C} 57 \mathrm{Bl} / 6$ and MX1-Cre mice were obtained from The Jackson Laboratory. Dyrk1a C57Bl/6 mutant mice have been described previously (17). Genotyping of Dyrk1a floxed or WT alleles was performed using PCR primers (forward, 5'-ATTACCTGGAGAAGAGGGCAAG-3' and reverse, 5'-TTCTTATGACTGGAATCGTCCC-3'). For xenograft experiments, NOD.Cg-Prkdc scid Il2rg ${ }^{\text {tmlWjl }}$ SzJ (NSG) mice were purchased from The Jackson Laboratory. Mice were maintained in specific pathogen-free conditions. Littermate controls were used for analysis in each cohort. Animals were backcrossed at least 10 generations. Male and female mice between birth and 1 year of age were studied.

Animal studies were performed to determine the genetic requirement of Dyrk1a in murine models of B-ALL, the on-target effects of EHT 1610 and AS1842856, and the efficacy of EHT 1610 and AS1842856 in treating mouse models of B-ALL. The sample size of the animal experiments, the specific transplantation protocols, and the dosing are described in the figure legends and in the appropriate 
Methods sections, respectively. For all in vivo experiments, mice were randomly assigned to transplant groups (genetic models) or treatment groups (inhibitor studies). All experiments were performed in an unblinded manner.

Murine model of $B C R-A B L^{+} B-A L L$. Bone marrow was collected from 4- to -6-week-old female C57BL/6 mice with Dyrk1 $1 a^{f / f l}$ or

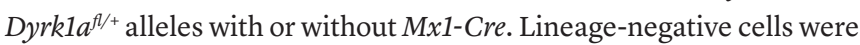
negatively selected from suspension of bone marrow cells using the EasySep Kit (STEMCELL Technologies) and transduced with concentrated retrovirus (MIGR1-BCR-ABL [p190]) as described below. Transduction was performed at $2500 \mathrm{rpm}(700 \mathrm{~g})$ for 90 minutes at $32^{\circ} \mathrm{C}$. Cells were then incubated at $37^{\circ} \mathrm{C}$ for an additional $4-6$ hours before replating in fresh medium OP9 stromal cells and then assayed for GFP and CD19 positivity by flow cytometry at $24-48$ hours and collected by filtration through a $40 \mu \mathrm{M}$ filter. Recipient mice were irradiated (900 cGy) and, within 4-24 hours, transplanted with $2 \times 10^{6}$ $\mathrm{GFP}^{+} \mathrm{CD} 19^{+}$cells in the presence of support cells from WT mice for primary transplantation. Once moribund, the mice were euthanized, and bone marrow and splenic cells were harvested and passed through a $70 \mu \mathrm{M}$ filter. Secondary transplantations were performed in irradiated mice by injection of $5 \times 10^{4} \mathrm{GFP}^{+} \mathrm{CD} 19^{+}$cells in the presence of support cells. To induce Cre expression in the murine model of B-ALL, animals received intraperitoneal (i.p.) injections of pI:pC ( $15 \mu \mathrm{g} / \mathrm{g}$ body weight; InvivoGen) every other day for a total of 4 injections. Injections began once $1 \%$ or more of peripheral blood $\mathrm{GFP}^{+} \mathrm{CD} 19^{+}$cells were detected by flow cytometry.

Inhibitors. The detailed synthesis of EHT 1610 has been described previously (28-30). EHT 1610 was obtained in 9 steps from 5-nitroanthranilonitrile in an overall yield of $14 \%$; its percentage of purity was estimated to be greater than 98\%. AS1842856 (S8222) and C188-9 (S8605) were purchased from SelleckChem; each batch was determined to have greater than $99 \%$ purity. The synthesis, characterization, and in vivo pharmacokinetics of AS1842856 and C188-9 are have been previously described in detail (refs. 48,61 , respectively). Batches were tested for recapitulation of the expected potency and phenotypes before use in experiments.

In vitro EHT 1610, AS1842856, and C188-9 treatment. Pre-B cells and B-ALL cell lines were treated with $2.5 \mu \mathrm{M}$ EHT 1610 or $100 \mathrm{nM}$ AS1842856 for inhibition assays unless otherwise indicated. For IC $_{50}$ calculations, EHT 1610, AS1842856, or C188-9 was added to cells in increasing concentrations in a white, opaque 96-well plate. Cells were incubated with EHT 1610 for 3 days, AS1842856 for 2 days, or C188-9 for 5 days. ATP levels were assayed by CellTiterGlo 2.0 Kit (Promega) following the manufacturer's protocol. For combination index (CI) assays, $\mathrm{IC}_{50}$ values were calculated as described above; multiples of $\mathrm{IC}_{50}$ values (Supplemental Table 3) for drugs were added to the cells for 2 days, and ATP levels were assayed using a CellTiterGlo 2.0 Kit. Calculation of combination indices was performed using the median-effect principle of Chou and Talalay (CompuSyn software) (83). A heatmap was generated using Morpheus (Broad Institute, https:// software.broadinstitute.org/morpheus/).

Drug responses were assessed in primary ALL cells cocultured on human telomerase reverse transcriptase-immortalized (hTERTimmortalized) primary bone marrow mesenchymal stromal cells (MSCs) in 384-well plates (Greiner, REF781090) as previously described (84). Briefly, $2.5 \times 10^{3}$ MSCs per well were plated in $30 \mu \mathrm{L}$ AIM-V medium 24 hours before addition of $2 \times 10^{4}$ to $3 \times 10^{4}$ ALL cells in $27.5 \mu \mathrm{L}$ medium recovered from cryopreserved samples. After 24 hours, drugs were added in serial dilutions using a Tecan D300 robot. Cell viability was assessed after 72 hours of drug incubation with the CyQUANT Direct Cell Proliferation Assay (Life Technologies, Thermo Fisher Scientific). Subsequently, automated imaging was performed using the ImageXpress Micro microscope (Molecular Devices). Images were processed using CellProfiler software (Broad Institute), and cells were classified and counted using Advanced Cell Classifier software (ETH Zürich, CH/Biological Research Centre, Szeged, Hungary) using an algorithm based on linear logistic regression models. For drug response quantification and statistical analysis, a fitting routine based on the 4-parameter log-logistic function (R package drc, version 2.3-96) was used. Data were normalized against DMSO-treated samples, and outliers were detected and removed prior to curve fitting by detection of local slope changes with a linear fit. Nonconvergent cases were identified on the basis of linear fit parameters. The R codes are available at https://github.com/pampernickel/drTools.

Flow cytometry and cell sorting. Flow cytometric analysis was performed on an LSR II Flow Cytometer (BD) and analyzed with FlowJo software (Tree Star). For B cell differentiation, single-cell suspensions of BMMCs were prepared and stained in FACS buffer (PBS plus 3\% FBS and 2 mM EDTA) containing fluorochrome-conjugated antibodies for surface markers, including B220, CD43, IgM, and CD19. CellTrace Violet analysis was performed according to the manufacturer's protocol (Thermo Fisher Scientific). For apoptotic cell staining, cell pellets were prepared, resuspended in annexin V binding buffer (BD), and stained for annexin V. For xenografts, single-cell suspensions of PBMCs were prepared and stained in FACS buffer containing fluorochrome-conjugated antibodies for surface markers, including human CD19 (hCD19) and hCD45. Cell-cycle analysis was performed as previously described (17).

For intracellular staining, cells were stained for surface markers, washed with FACS buffer, fixed for 30 minutes with 1:1 IC Fixation buffer (eBioscience) in FACS buffer, washed, permeabilized with ice-cold Perm Buffer III (BD), washed again, and then stained with fluorochrome-conjugated antibodies against $\gamma-\mathrm{H} 2 \mathrm{AX}$ or with an unconjugated antibody against pCDK1 Y15 followed by a fluorochrome-conjugated secondary antibody against rabbit epitope. Cell sorting was performed with FACSMelody (BD). For pre-B cell sorting, large and small pre-B populations were sorted as previously described (17). For murine B-ALL analysis, $\mathrm{GFP}^{+}$cells were sorted from mouse bone marrow. The antibodies used are listed in Supplemental Table 9.

Xenograft models of $B-A L L$. NSG mice received transplants at 12-14 weeks of age. For cell line xenografts, nonirradiated NSG mice were transplanted with $1 \times 10^{6} \mathrm{MHH}-\mathrm{CALL}-4$ or Nalm- 6 cells. After detection of $1 \%$ or more of peripheral blood hCD19+hCD $45^{+}$cells, treatment was performed by i.p. injection of $20 \mathrm{mg} / \mathrm{kg}$ EHT 1610 b.i.d. (5 days on, 2 days off) or $10 \mathrm{mg} / \mathrm{kg} /$ day AS1842856 daily, respectively, or vehicle (10\% ethanol, 50\% PEG-400, and 5\% Tween-80) for 2 weeks.

For PDXs, NSG mice were sublethally irradiated (150 cGy) and transplanted with $1 \times 10^{6}$ cells. DS- and HeH-ALL PDX models have been previously described (22). Mice were euthanized when moribund, and splenic cells were obtained and analyzed for the presence of hCD19+hCD $45^{+}$cells. Cells were either cultured ex vivo for $\mathrm{IC}_{50}$ experiments or transplanted into secondary recipients at $2 \times 10^{5}$ cells per recipient. In luciferase-expressing models of ALL (DS-ALL-03 or HeH-ALL-09), treatment began once $10^{7} \mathrm{p} / \mathrm{s}$ was detected on the IVIS 
Spectrum imaging system (PerkinElmer). DS-ALL-03 recipients were treated for 3 weeks with vehicle, $20 \mathrm{mg} / \mathrm{kg}$ EHT 1610 b.i.d. (5 days on,

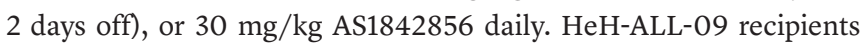
were treated for 3 weeks with vehicle, $20 \mathrm{mg} / \mathrm{kg}$ EHT 1610 b.i.d. (5 days on, 2 days off), or $30 \mathrm{mg} / \mathrm{kg}$ AS1842856 daily. Dosing regimens were based on empiric data from C57BL/6 mice (Supplemental Figure 7) for EHT 1610 and on previously published data (79) for AS1842856.

Data and materials availability. All data associated with this study are presented here or in the supplemental materials.

Statistics. All statistical analysis was performed using GraphPad Prism 6 software for Mac (GraphPad Software). Comparison of 2 means was analyzed by unpaired, 2-tailed Student's $t$ test. When multiple comparisons were necessary, 1-way or 2-way ANOVA with post hoc Bonferroni's correction was used. Survival analysis was performed using the Kaplan-Meier method with log-rank (Mantel-Cox) comparison of survival curves. $\mathrm{IC}_{50}$ curves were nonlinearly fit with variable slope (4 parameters). Unless otherwise noted, data are summarized as the mean \pm SD. The significance threshold was set at a $P$ value of less than 0.05 .

Study approval. All animal studies were approved by the IACUC of Northwestern University. Specimens from patients with B-ALL or T-ALL were obtained after informed consent, with approval of the IRBs of the Canton of Zurich and Northwestern University. All study participants were deidentified.

\section{Author contributions}

RSB, MR, PL, APL, AS, QW, PS, BHY, YCT, SJ, BB, AS, APB, EH, and YAG performed the experiments and analyzed the data. $C F$, $\mathrm{TB}, \mathrm{NH}, \mathrm{SI}$, and JPB analyzed the data. BJT and MV provided critical insights. SM and JDC designed the experiments and analyzed the data. All authors contributed to the writing of the manuscript.

\section{Acknowledgments}

This work was supported by the NIH (CA101774, to JDC, and CA211534, to QW). The content is solely the responsibility of the authors and does not necessarily represent the official views of the NIH. Additional support was provided by the Samuel Waxman Cancer Research Foundation, the Rally Foundation, the Leukemia
Research Foundation (to SM, 2012), the European Hematology Association (EHA) (to SM, 2013), the Fondation Lejeune (grant no. 1806), the Children's Leukaemia and Cancer Research Foundation (CLCRF), and the United States - Israel Binational Science Foundation (BSF). RSB received support from the Alpha Omega Alpha Carolyn L. Kuckein Award, the American Society of Hematology (ASH) HONORS (Hematology Opportunities for the Next Generation of Research Scientists) Award, and a Howard Hughes Medical Institute (HHMI) Medical Research Fellowship. APL was supported by the Cancéropôle Ile-de-France. PL received support from the Junior Board of the Children's Research Fund. SI was supported by the Israel Cancer Research Foundation, the Israel Science Foundation, and the Ministry of Health of Israel. JPB was supported by the Swiss National Research Foundation and the Clinical Research Priority Program of the University of Zurich. SM is supported by a Fellowship from the Cancer Council Western Australia (CCWA). CF and TB thank Normandie University and LABEX SynOrg (ANR-11-LABX-0029) for financial support. Proteomics services were provided by the Northwestern University Proteomics Core Facility (supported by National Cancer Institute [NCI], NIH, Cancer Center Support Grants [CCSGs] P30 CA060553 and P41 GM108569) as well as the University of Michigan Proteomics Facility. Imaging was performed at the Northwestern University Center for Advanced Microscopy, which is supported by the NCI, CCSG grant P30 CA060553. Multiphoton microscopy was performed on a Nikon A1R multiphoton microscope, acquired through the support of NIH grant 1S10OD010398-01. The authors thank Eric Delabesse and Diana Fan for their advice and Johanna Melo-Cardenas for technical assistance. The graphical abstract was created with BioRender.com.

Address correspondence to: John D. Crispino, St. Jude Children's Research Hospital, 262 Danny Thomas Place, MS341, Memphis, Tennessee 38105, USA. Phone: 901.595.7511; Email: j-crispino@ northwestern.edu or john.crispino@stjude.org.

RSB's present address is: Department of Medicine, Hospital of the University of Pennsylvania, Philadelphia, Pennsylvania, USA.
1. Ward E, DeSantis C, Robbins A, Kohler B, Jema A. Childhood and adolescent cancer statistics, 2014. CA Cancer JClin. 2014;64(2):83-103.

2. Hunger SP, et al. Children's Oncology Group's 2013 blueprint for research: acute lymphoblastic leukemia. Pediatr Blood Cancer. 2013;60(6):957-963.

3. Hasle H, Clemmensen IH, Mikkelsen M. Risks of leukaemia and solid tumours in individuals with Down's syndrome. Lancet. 2000;355(9199):165-169.

4. Buitenkamp TD, et al. Acute lymphoblastic leukemia in children with Down syndrome: a retrospective analysis from the Ponte di Legno study group. Blood. 2014;123(1):70-77.

5. Lee P, Bhansali R, Izraeli S, Hijiya N, Crispino JD. The biology, pathogenesis and clinical aspects of acute lymphoblastic leukemia in children with Down syndrome. Leukemia. 2016;30(9):1816-1823.
6. Lane AA, et al. Triplication of a 21q22 region contributes to B cell transformation through HMGN1 overexpression and loss of histone H3 Lys27 trimethylation. Nat Genet. 2014;46(6):618-623.

7. Malinge $\mathrm{S}$, et al. Increased dosage of the chromosome 21 ortholog Dyrk1a promotes megakaryoblastic leukemia in a murine model of Down syndrome. J Clin Invest. 2012;122(3):948-962.

8. Li Y, et al. Constitutional and somatic rearrangement of chromosome 21 in acute lymphoblastic leukaemia. Nature. 2014;508(7494):98-102.

9. Rivera-Munoz P, et al. Partial trisomy 21 contributes to T-cell malignancies induced by JAK3activating mutations in murine models. Blood Adv. 2018;2(13):1616-1627.

10. Laurent AP, Kotecha RS, Malinge S. Gain of chromosome 21 in hematological malignancies: lessons from studying leukemia in children with Down syndrome. Leukemia. 2020;34(8):1984-1999.

11. Abbassi R, Johns TG, Kassiou M, Munoz L. DYR-
K1A in neurodegeneration and cancer: molecular basis and clinical implications. Pharmacol Ther. 2015;151:87-98.

12. Dang T, et al. Autism-associated Dyrk1a truncation mutants impair neuronal dendritic and spine growth and interfere with postnatal cortical development. Mol Psychiatry. 2018;23(3):747-758.

13. Wiseman FK, et al. A genetic cause of Alzheimer disease: mechanistic insights from Down syndrome. Nat Rev Neurosci. 2015;16(9):564-574.

14. Barallobre MJ, et al. DYRK1A promotes dopaminergic neuron survival in the developing brain and in a mouse model of Parkinson's disease. Cell Death Dis. 2014;5:e1289.

15. Litovchick L, Florens LA, Swanson SK, Washburn MP, DeCaprio JA. DYRK1A protein kinase promotes quiescence and senescence through DREAM complex assembly. Genes Dev. 2011;25(8):801-813.

16. Soppa U, Schumacher J, Florencio Ortiz V, 
Pasqualon T, Tejedor FJ, Becker W. The Down syndrome-related protein kinase DYRK1A phosphorylates p27(Kip1) and cyclin D1 and induces cell cycle exit and neuronal differentiation. Cell Cycle. 2014;13(13):2084-2100.

17. Thompson BJ, et al. DYRK1A controls the transition from proliferation to quiescence during lymphoid development by destabilizing cyclin D3. JExp Med. 2015;212(6):953-970.

18. Chen JY, Lin JR, Tsai FC, Meyer T. Dosage of Dyrk1a shifts cells within a p21-cyclin D1 signaling map to control the decision to enter the cell cycle. Mol Cell. 2013;52(1):87-100.

19. Di Vona C, et al. Chromatin-wide profiling of DYRK1A reveals a role as a gene-specific RNA polymerase II CTD kinase. Mol Cell. 2015;57(3):506-520.

20. Ding $S$, et al. Regulation of alternative splicing of tau exon 10 by $9 \mathrm{G} 8$ and Dyrk1A. Neurobiol Aging. 2012;33(7):1389-1399.

21. Barretina J, et al. The Cancer Cell Line Encyclopedia enables predictive modelling of anticancer drug sensitivity. Nature. 2012;483(7391):603-607.

22. Laurent AP, et al. Constitutive activation of RAS/MAPK pathway cooperates with trisomy 21 is therapeutically exploitable in down syndrome B-cell leukemia. Clin Cancer Res. 2020;26(13):3307-3318.

23. Zhou X, et al. Exploring genomic alteration in pediatric cancer using ProteinPaint. Nat Genet. 2016;48(1):4-6.

24. Rothenberg EV. Transcriptional control of early $\mathrm{T}$ and B cell developmental choices. Annu Rev Immunol. 2014;32:283-321.

25. Sitz JH, Tigges M, Baumgärtel K, Khaspekov LG, Lutz B. Dyrk1A potentiates steroid hormone-induced transcription via the chromatin remodeling factor Arip4. Mol Cell Biol. 2004;24(13):5821-5834.

26. Yang EJ, Ahn YS, Chung KC. Protein kinase Dyrk1 activates cAMP response elementbinding protein during neuronal differentiation in hippocampal progenitor cells. J Biol Chem. 2001;276(43):39819-39824.

27. Yin X, et al. Dual-specificity tyrosine phosphorylation-regulated kinase 1A (Dyrk1A) modulates serine/arginine-rich protein 55 (SRp55)-promoted Tau exon 10 inclusion. J Biol Chem. 2012;287(36):30497-30506.

28. Chaikuad A, et al. An unusual binding model of the methyl 9-anilinothiazolo[5,4-f] quinazoline-2-carbimidates (EHT 1610 and EHT 5372) confers high selectivity for dual-specificity tyrosine phosphorylation-regulated kinases. J Med Chem. 2016;59(22):10315-10321.

29. Foucourt A, et al. Design and synthesis of thiazolo[5,4-f] quinazolines as DYRK1A inhibitors, part II. Molecules. 2014;19(10):15411-15439.

30. Foucourt A, et al. Design and synthesis of thiazolo[5,4-f] quinazolines as DYRK1A inhibitors, part I. Molecules. 2014;19(10):15546-15571.

31. Jarhad DB, Mashelkar KK, Kim HR, Noh M, Jeong LS. Dual-specificity tyrosine phosphorylation-regulated kinase 1A (DYRK1A) inhibitors as potential therapeutics. JMed Chem. 2018;61(22):9791-9810.

32. Göckler N, Jofre G, Papadopoulos C, Soppa $\mathrm{U}$, Tejedor FJ, Becker W. Harmine specifi- cally inhibits protein kinase DYRK1A and interferes with neurite formation. FEBS J. 2009;276(21):6324-6337.

33. Ogawa Y, et al. Development of a novel selective inhibitor of the Down syndrome-related kinase Dyrk1A. Nat Commun. 2010;1:86.

34. Lutz C, et al. Quiescent leukaemic cells account for minimal residual disease in childhood lymphoblastic leukaemia. Leukemia. 2013;27(5):1204-1207.

35. Ebinger S, et al. Characterization of rare, dormant, and rherapy-resistant cells in acute lymphoblastic leukemia. Cancer Cell. 2016;30(6):849-862.

36. Szklarczyk D, et al. STRING v10: protein-protein interaction networks, integrated over the tree of life. Nucleic Acids Res. 2015;43(Database issue):D447-D452.

37. Kanehisa M, Sato Y, Kawashima M, Furumichi M, Tanabe M. KEGG as a reference resource for gene and protein annotation. Nucleic Acids Res. 2016;44(D1):D457-D462.

38. Kanehisa M, Furumichi M, Tanabe M, Sato Y, Morishima K. KEGG: new perspectives on genomes, pathways, diseases and drugs. Nucleic Acids Res. 2017;45(D1):D353-D361.

39. Kanehisa M, Goto S. KEGG: Kyoto encyclopedia of genes and genomes. Nucleic Acids Res. 2000;28(1):27-30.

40. Woods YL, et al. The kinase DYRK1A phosphorylates the transcription factor FKHR at Ser329 in vitro, a novel in vivo phosphorylation site. Biochem J. 2001;355(Pt 3):597-607.

41. Kurabayashi N, Nguyen MD, Sanada K. DYRK1A overexpression enhances STAT activity and astrogliogenesis in a Down syndrome mouse model. EMBO Rep. 2015;16(11):1548-1562.

42. The Gene Ontology Consortium. Expansion of the Gene Ontology knowledgebase and resources. Nucleic Acids Res. 2017;45(D1):D331-D338.

43. Ashburner M, et al. Gene ontology: tool for the unification of biology. The Gene Ontology Consortium. Nat Genet. 2000;25(1):25-29.

44. Dengler HS, et al. Distinct functions for the transcription factor Foxo1 at various stages of B cell differentiation. Nat Immunol. 2008;9(12):1388-1398.

45. Huang H, Tindall DJ. Dynamic FoxO transcription factors. JCell Sci. 2007;120 (pt 15):2479-2487.

46. Matsuzaki H, Daitoku H, Hatta M, Tanaka K, Fukamizu A. Insulin-induced phosphorylation of FKHR (Foxo1) targets to proteasomal degradation. Proc Natl Acad Sci U S A. 2003;100(20):11285-11290.

47. van der Horst A, Burgering BM. Stressing the role of FoxO proteins in lifespan and disease. Nat Rev Mol Cell Biol. 2007;8(6):440-450.

48. Nagashima T, et al. Discovery of novel forkhead box $\mathrm{O} 1$ inhibitors for treating type 2 diabetes: improvement of fasting glycemia in diabetic db/db mice. Mol Pharmacol. 2010;78(5):961-970.

49. Amin RH, Schlissel MS. Foxo1 directly regulates the transcription of recombination-activating genes during B cell development. Nat Immunol. 2008;9(6):613-622.

50. Wang XW, et al. GADD45 induction of a G2/M cell cycle checkpoint. Proc Natl Acad Sci U S A. 1999;96(7):3706-3711.
51. Yang R, Rincon M. Mitochondrial Stat3, the need for design thinking. Int J Biol Sci. 2016;12(5):532-544.

52. Wen Z, Zhong Z, Darnell JE. Maximal activation of transcription by Stat 1 and Stat 3 requires both tyrosine and serine phosphorylation. Cell. 1995;82(2):241-250.

53. Hazan-Halevy I, et al. STAT3 is constitutively phosphorylated on serine 727 residues, binds DNA, and activates transcription in CLL cells. Blood. 2010;115(14):2852-2863.

54. Huang G, Yan H, Ye S, Tong C, Ying QL. STAT3 phosphorylation at tyrosine 705 and serine 727 differentially regulates mouse ESC fates. Stem Cells. 2014;32(5):1149-1160.

55. Gough DJ, Corlett A, Schlessinger K, Wegrzyn J, Larner AC, Levy DE. Mitochondrial STAT3 supports Ras-dependent oncogenic transformation. Science. 2009;324(5935):1713-1716.

56. Wegrzyn J, et al. Function of mitochondrial Stat3 in cellular respiration. Science. 2009;323(5915):793-797.

57. Zhang Q, et al. Mitochondrial localized Stat3 promotes breast cancer growth via phosphorylation of serine 727. J Biol Chem. 2013;288(43):31280-31288.

58. Mantel C, et al. Mouse hematopoietic celltargeted STAT3 deletion: stem/progenitor cell defects, mitochondrial dysfunction, ROS overproduction, and a rapid aging-like phenotype. Blood. 2012;120(13):2589-2599.

59. Sarafian TA, et al. Disruption of astrocyte STAT3 signaling decreases mitochondrial function and increases oxidative stress in vitro. PLoS One. 2010;5(3):e9532.

60. Szczepanek K, et al. Mitochondrial-targeted Signal transducer and activator of transcription 3 (STAT3) protects against ischemia-induced changes in the electron transport chain and the generation of reactive oxygen species. J Biol Chem. 2011;286(34):29610-29620.

61. Bharadwaj U, et al. Small-molecule inhibition of STAT3 in radioresistant head and neck squamous cell carcinoma. Oncotarget. 2016;7(18):26307-26330.

62. Di JX, Zhang HY. C188-9, a small-molecule STAT3 inhibitor, exerts an antitumor effect on head and neck squamous cell carcinoma. Anticancer Drugs. 2019;30(8):846-853.

63. Lewis KM, et al. Small-molecule targeting of signal transducer and activator of transcription (STAT) 3 to treat non-small cell lung cancer. Lung Cancer. 2015;90(2):182-190.

64. Bai L, et al. A potent and selective smallmolecule degrader of STAT3 achieves complete tumor regression in vivo. Cancer Cell. 2019;36(5):498-511.e17.

65. Zhou H, et al. Structure-based discovery of SD-36 as a potent, selective, efficacious PROTAC degrader of STAT3 protein. JMed Chem. 2019;62(24):11280-11300.

66. Peeters MA, Rethore MO, Lejeune J. In vivo folic acid supplementation partially corrects in vitro methotrexate toxicity in patients with Down syndrome. Br J Haematol. 1995;89(3):678-680.

67. Kalwinsky DK, et al. Clinical and biological characteristics of acute lymphocytic leukemia in children with Down syndrome. Am JMed Genet 
Suppl.1990;7:267-271.

68. Löwenberg B, et al. Effect of priming with granulocyte colony-stimulating factor on the outcome of chemotherapy for acute myeloid leukemia. N Engl JMed. 2003;349(8):743-752.

69. Yin X, et al. Dyrk1A overexpression leads to increase of 3R-tau expression and cognitive deficits in Ts65Dn Down syndrome mice. Sci Rep. 2017;7(1):619.

70. Guedj F, et al. Green tea polyphenols rescue of brain defects induced by overexpression of DYRK1A. PLoS One. 2009;4(2):e4606.

71. Wetzler M, et al. Additional cytogenetic abnormalities in adults with Philadelphia chromosome-positive acute lymphoblastic leukaemia: a study of the Cancer and Leukaemia Group B. Br J Haematol. 2004;124(3):275-288.

72. Van Der Heide LP, Hoekman MF, Smidt MP. The ins and outs of FoxO shuttling: mechanisms of FoxO translocation and transcriptional regulation. Biochem J. 2004;380(pt 2):297-309.
73. Arron JR, et al. NFAT dysregulation by increased dosage of DSCR1 and DYRK1A on chromosome 21. Nature. 2006;441(7093):595-600.

74. Pozo N, et al. Inhibition of DYRK1A destabilizes EGFR and reduces EGFR-dependent glioblastoma growth. J Clin Invest. 2013;123(6):2475-2487.

75. Lee SB, et al. An ID2-dependent mechanism for VHL inactivation in cancer. Nature. 2016;529(7585):172-177.

76. Trinh DL, et al. Analysis of FOXO1 mutations in diffuse large B-cell lymphoma. Blood. 2013;121(18):3666-3674.

77. Kabrani E, et al. Nuclear FOXO1 promotes lymphomagenesis in germinal center B cells. Blood. 2018;132(25):2670-2683.

78. Sykes SM, et al. AKT/FOXO signaling enforces reversible differentiation blockade in myeloid leukemias. Cell. 2011;146(5):697-708.

79. Wang F, et al. Tight regulation of FOXO1 is essential for maintenance of B-cell precursor acute lymphoblastic leukemia. Blood.
2018;131(26):2929-2942.

80. Herold T, et al. Adults with Philadelphia chromosome-like acute lymphoblastic leukemia frequently have IGH-CRLF2 and JAK2 mutations, persistence of minimal residual disease and poor prognosis. Haematologica. 2017;102(1):130-138.

81. Roberts KG, et al. Targetable kinase-activating lesions in Ph-like acute lymphoblastic leukemia. N EnglJMed. 2014;371(11):1005-1015.

82. Engelman JA, Settleman J. Acquired resistance to tyrosine kinase inhibitors during cancer therapy. Curr Opin Genet Dev. 2008;18(1):73-79.

83. Chou TC, Talalay P. Quantitative analysis of dose-effect relationships: the combined effects of multiple drugs or enzyme inhibitors. $A d v$ Enzyme Regul. 1984;22:27-55.

84. Fischer U, et al. Genomics and drug profiling of fatal TCF3-HLF-positive acute lymphoblastic leukemia identifies recurrent mutation patterns and therapeutic options. Nat Genet. 2015;47(9):1020-1029. 